\title{
Self-Assembly of Bidentate Ligands for Combinatorial Homogeneous Catalysis Asymmetric Rhodium-Catalyzed Hydrogenation
}

\author{
Bernhard Breit,* Martine Weis, Christoph Waloch and Wolfgang Seiche \\ Institut für Organische Chemie und Biochemie, Albert-Ludwigs-Universität Freiburg, Germany
}

\section{Supplementary Material}

(24 pages)

I General S-1

II Experimental procedures and characterizations $\quad$ S-3

$1 \quad$ Preparation of aminopyridinyl phosphines $\quad$ S-3

$2 \quad$ Preparation of aminopyridinyl phosphonites $\quad$ S-8

$3 \quad$ Preparation of isoquinolinyl phosphonites $\quad$ S-12

$\begin{array}{ll}\text { III General procedure for asymmetric hydrogenation } & \text { S-16 }\end{array}$

$\begin{array}{ll}\text { IV Heterodimeric platinum complexes } & \text { S-17 }\end{array}$

V Heterodimeric cationic rhodium complexes $\quad$ S-19

$\begin{array}{llr}\text { VI Literature } & \text { S-24 }\end{array}$

\section{General}

Bisdiethylamino-chloro-phosphine, ${ }^{[1]}$ 2-(trimethylsilyl)ethanol, ${ }^{[2]}$ the BINOL-derivatives,${ }^{[3]} 2$ amino-6-bromopyridine, ${ }^{[4]} \quad$ 2-bromo-6- $N$-pivaloylaminopyridine ${ }^{[5]}$ and 1,3-dibromoisoquinoline $^{[6]}$ were synthesized according to literature procedures. The unsymmetrical disubstituted chlorophosphines can be prepared according to literature. ${ }^{[7]}$ Methyl-2acetamidoacrylate and dimethylitaconate were purchased from Aldrich and Jansen Chimica, methyl- $\alpha$-acetylamino cinnamate ${ }^{[8]}$ was synthesized according to literature procedures.

All reactions were carried out in dried glassware under an argon atmosphere 5.0 (SüdwestGas). Air and moisture sensitive liquids and solutions were transferred via syringe. All reagents were obtained commercially unless otherwise noted. All solvents were dried and distilled by standard procedures. Organic solutions were concentrated under reduced pressure by rotary evaporation. Chromatographic purification of products was accomplished using flash chromatography ${ }^{[9]}$ on a Merck silica gel Si $60^{\circledR}$ (200-400 mesh). 
Nuclear magnetic resonance spectra were acquired on a Varian Mercury spectrometer (300 MHz, $121 \mathrm{MHz}$ and $75 \mathrm{MHz}$ for ${ }^{1} \mathrm{H},{ }^{31} \mathrm{P}$ and ${ }^{13} \mathrm{C}$ respectively), on a Bruker AMX 400 (400 MHz, $162 \mathrm{MHz}$ and $100 \mathrm{MHz}$ for ${ }^{1} \mathrm{H},{ }^{31} \mathrm{P}$ and ${ }^{13} \mathrm{C}$ respectively) and on a Bruker DRX $500\left(500 \mathrm{MHz}, 202 \mathrm{MHz}\right.$ and $125 \mathrm{MHz}$ for ${ }^{1} \mathrm{H},{ }^{31} \mathrm{P}$ and ${ }^{13} \mathrm{C}$ respectively) and are referenced according to residual protio solvent signals. Data for ${ }^{1} \mathrm{H}-\mathrm{NMR}$ are recorded as follows: chemical shift ( $\delta$ in ppm), multiplicity (s, singlet; br s, broad singlet; d, doublet; t, triplet; q, quartet; m, multiplet; $\mathrm{p}$, pseudo), coupling constant $(\mathrm{Hz})$, integration. Data for ${ }^{13} \mathrm{C}-\mathrm{NMR}$ are reported in terms of chemical shift ( $\delta$ in ppm), multiplicity (if not a singlet), coupling constant $(\mathrm{Hz})$.

High-resolution mass spectra were obtained on a Finnigan MAT 8200 instrument and ESI mass spectrometry was performed on a Finnigan LCQ Advantage. Elementary analysis was performed on an elementar vario (Fa. Elementar Analysensysteme $\mathrm{GmbH}$ ). Optical rotations were measured on a Perkin-Elmer 241 polarimeter.

Separation of the enantiomers was achieved on a Chiralpak AD-H column using a Knaur K$2501 \mathrm{UV}$-detector. The enantiomeric excess of the triarylphosphine ligands was determined by HPLC using a Chiralpak AD-H column.

Hydrogenation experiments were performed following the general procedure using hydrogen gas 5.0 (Südwest-Gas). 


\section{Experimental procedures and characterizations}

$1 \quad$ Preparation of aminopyridinyl phosphines

(rac)-6-(o-Anisylphenylphosphino)-2-pivaloylaminopyridine (4a)<smiles>COc1ccccc1P(c1ccccc1)c1cccc(NC(=O)C(C)(C)C)n1</smiles>

$\mathrm{C}_{23} \mathrm{H}_{25} \mathrm{~N}_{2} \mathrm{O}_{2} \mathrm{P}$

Mol. Wt.: 392.43

To a solution of $2.42 \mathrm{~g}$ 2-bromo-6- $N$-pivaloylaminopyridine (9.4 mmol, 0.8 eq.) in $30 \mathrm{ml}$ THF was added at $-100^{\circ} \mathrm{C} 11.7 \mathrm{ml} n$-butyl-lithium ( $1.60 \mathrm{M}$ in hexane, $18.8 \mathrm{mmol}, 1.6$ eq.) during $20 \mathrm{~min}$. The mixture was stirred for $1 \mathrm{~h}$, then a solution of $2.95 \mathrm{~g} o$-anisylchlorophosphine (11.8 mmol, 1.0 eq.) in $10 \mathrm{ml} \mathrm{THF}$ was added slowly. After stirring $30 \mathrm{~min}$ at $-100^{\circ} \mathrm{C}$, the yellow solution was allowed to warm to room temperature and stirred $24 \mathrm{~h}$ at this temperature. The solvent was removed under reduced pressure and the residue was filtrated through a plug of silica to remove salts. The filtrate was concentrated in vacuo and purified via flash chromatography (Cy:EtOAc 10:1) to yield $1.84 \mathrm{~g}$ of the title compound 4a (4.7 $\mathrm{mmol}, 50 \%)$ as a white foam.

${ }^{1}$ H-NMR (400 MHz, $\left.\mathrm{C}_{6} \mathrm{D}_{6}\right): \delta 8.51(\mathrm{~d}, J=8.2 \mathrm{~Hz}, 1 \mathrm{H}), 7.88$ (br s, $\left.1 \mathrm{H}\right), 7.52-7.48(\mathrm{~m}, 2 \mathrm{H})$, 7.12-7.05 (m, 5H), 7.04-7.00 (m, 1H), 6.82 (d, $J=7.3 \mathrm{~Hz}, 1 \mathrm{H}), 6.74(\mathrm{dd}, J=7.3,7.3 \mathrm{~Hz}, 1 \mathrm{H})$, $6.50(\mathrm{dd}, J=8.2,4.7 \mathrm{~Hz}, 1 \mathrm{H}), 3.19$ (s, 3H), $0.86(\mathrm{~s}, 9 \mathrm{H}) ;{ }^{13} \mathbf{C}-\mathbf{N M R}\left(100 \mathrm{MHz}, \mathrm{C}_{6} \mathrm{D}_{6}\right): \delta$ $176.5,162.7\left(\mathrm{~d}, J_{\mathrm{C}, \mathrm{P}}=4.3 \mathrm{~Hz}\right), 161.9\left(\mathrm{~d}, J_{\mathrm{C}, \mathrm{P}}=14.5 \mathrm{~Hz}\right), 152.6\left(\mathrm{~d}, J_{\mathrm{C}, \mathrm{P}}=14.5 \mathrm{~Hz}\right), 137.9$, $136.7\left(\mathrm{~d}, J_{\mathrm{C}, \mathrm{P}}=11.6 \mathrm{~Hz}\right), 135.1\left(\mathrm{~d}, J_{\mathrm{C}, \mathrm{P}}=20.3 \mathrm{~Hz}\right), 134.3\left(\mathrm{~d}, J_{\mathrm{C}, \mathrm{P}}=2.9 \mathrm{~Hz}\right), 130.6,139.1$, $128.8\left(\mathrm{~d}, J_{\mathrm{C}, \mathrm{P}}=7.3 \mathrm{~Hz}\right), 128.0\left(\mathrm{~d}, J_{\mathrm{C}, \mathrm{P}}=21.8 \mathrm{~Hz}\right), 126.0\left(\mathrm{~d}, J_{\mathrm{C}, \mathrm{P}}=14.5 \mathrm{~Hz}\right), 123.8\left(\mathrm{~d}, J_{\mathrm{C}, \mathrm{P}}=\right.$ 11.6 Hz), 112.5, 110.7, 55.2, 39.5, 27.0; ${ }^{31}$ P-NMR (121 MHz, $\left.\mathrm{C}_{6} \mathrm{D}_{6}\right): \delta-14.2$; CHN: calcd. C: $70.39, \mathrm{H}: 6.42, \mathrm{~N}: 7.14$ found C: 70.16, H: 6.62, N: 6.97.

The enantiomers of $\mathbf{4 a}$ were separated via preparative chiral HPLC (Chiralpak AD-H, $n$ heptane: $i$-PrOH 100:5, rt, $12.0 \mathrm{ml} / \mathrm{min}, 295 \mathrm{~nm})$; (-)-enantiomer : $6.4 \mathrm{~min},[\alpha]_{\mathrm{D}}{ }^{21}=-22.8(\mathrm{c}=$ 0.98, $\left.\mathrm{CHCl}_{3}\right) ;(+)$-enantiomer: $11.9 \mathrm{~min},[\alpha]_{\mathrm{D}}^{21}=+22.6\left(\mathrm{c}=1.01, \mathrm{CHCl}_{3}\right)$.

(The analytical and spectroscopic data of the enantiomers correspond to the racemate) 


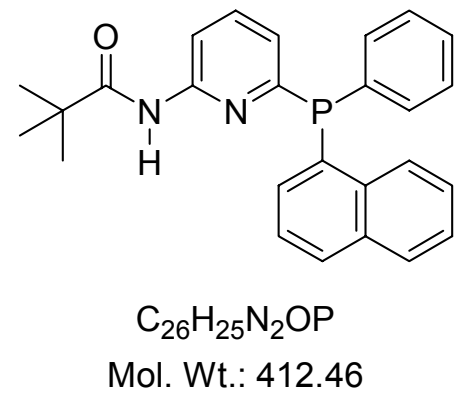

To a solution of $1.80 \mathrm{~g}$ 2-bromo-6- $N$-pivaloylaminopyridine ( $7.0 \mathrm{mmol}, 1.0$ eq.) in $30 \mathrm{ml} \mathrm{THF}$ was added at $-100^{\circ} \mathrm{C} 8.7 \mathrm{ml} n$-butyl-lithium (1.60 $\mathrm{M}$ in hexane, $14.0 \mathrm{mmol}, 2.0$ eq.) during $20 \mathrm{~min}$. The mixture was stirred for $1 \mathrm{~h}$, then a solution of $1.89 \mathrm{~g} 1$ naphtylphenylchlorophosphine (7.0 mmol, 1.0 eq.) in $10 \mathrm{ml}$ THF was slowly added. After stirring $30 \mathrm{~min}$ at $-100^{\circ} \mathrm{C}$, the yellow solution was allowed to warm to room temperature and stirred $12 \mathrm{~h}$ at this temperature. After addition of $30 \mathrm{ml}$ sat. aqueous $\mathrm{NaHCO}_{3}$ solution, the aqueous layer was extracted with EtOAc $(3 \times 20 \mathrm{ml})$, the combined organic layers were dried $\left(\mathrm{MgSO}_{4}\right)$ and concentrated. The crude product was purified via flash chromatography (Cy:EtOAc 10:1) to yield $1.50 \mathrm{~g}$ of the title compound $\mathbf{4 b}(3.6 \mathrm{mmol}, 52 \%)$ as a white foam. ${ }^{1}$ H-NMR $\left(500 \mathrm{MHz}, \mathrm{C}_{6} \mathrm{D}_{6}\right): \delta 8.77-8.75(\mathrm{~m}, 1 \mathrm{H}), 8.51$ (d, $\left.J=7.5 \mathrm{~Hz}, 1 \mathrm{H}\right), 7.92$ (br s, $\left.1 \mathrm{H}\right)$, 7.60-7.57 (m, 2H), 7.47-7.43 (m, 2H), $7.29(\mathrm{td}, J=5.6,1.1 \mathrm{~Hz}, 1 \mathrm{H}), 7.19-7.17(\mathrm{~m}, 2 \mathrm{H}), 7.11$ (t, $J=7.7 \mathrm{~Hz}, 1 \mathrm{H}), 7.05-7.04(\mathrm{~m}, 3 \mathrm{H}), 6.93(\mathrm{td}, J=7.6,1.9 \mathrm{~Hz}, 1 \mathrm{H}), 6.66(\mathrm{~d}, J=7.5 \mathrm{~Hz}, 1 \mathrm{H})$, $0.86(\mathrm{~s}, 9 \mathrm{H}) ;{ }^{13} \mathrm{C}-\mathbf{N M R}\left(125 \mathrm{MHz}, \mathrm{C}_{6} \mathrm{D}_{6}\right): \delta 176.5,161.9\left(\mathrm{~d}, J_{\mathrm{C}, \mathrm{P}}=6.4 \mathrm{~Hz}\right), 152.8\left(\mathrm{~d}, J_{\mathrm{C}, \mathrm{P}}=\right.$ $15.1 \mathrm{~Hz}), 138.2\left(\mathrm{~d}, J_{\mathrm{C}, \mathrm{P}}=1.5 \mathrm{~Hz}\right), 136.3\left(\mathrm{~d}, J_{\mathrm{C}, \mathrm{P}}=22.1 \mathrm{~Hz}\right), 136.0\left(\mathrm{~d}, J_{\mathrm{C}, \mathrm{P}}=10.9 \mathrm{~Hz}\right), 135.1$ $\left(\mathrm{d}, J_{\mathrm{C}, \mathrm{P}}=20.6 \mathrm{~Hz}\right), 134.4\left(J_{\mathrm{C}, \mathrm{P}}=15.1 \mathrm{~Hz}\right), 134.0\left(\mathrm{~d}, J_{\mathrm{C}, \mathrm{P}}=4.2 \mathrm{~Hz}\right), 132.9\left(\mathrm{~d}, J_{\mathrm{C}, \mathrm{P}}=1.2 \mathrm{~Hz}\right)$, 130.0, 129.3, 129.0, $128.9\left(\mathrm{~d}, J_{\mathrm{C}, \mathrm{P}}=7.3 \mathrm{~Hz}\right), 126.7\left(\mathrm{~d}, J_{\mathrm{C}, \mathrm{P}}=18.1 \mathrm{~Hz}\right), 126.6,126.4\left(\mathrm{~d}, J_{\mathrm{C}, \mathrm{P}}=\right.$ $1.5 \mathrm{~Hz}), 126.0\left(\mathrm{~d}, J_{\mathrm{C}, \mathrm{P}}=1.8 \mathrm{~Hz}\right), 124.5\left(\mathrm{~d}, J_{\mathrm{C}, \mathrm{P}}=11.8 \mathrm{~Hz}\right), 112.8,39.5,27.1 ;{ }^{31} \mathbf{P}-\mathbf{N M R}$ (162 MHz, $\left.\mathrm{C}_{6} \mathrm{D}_{6}\right): \delta-12.6$; CHN: calcd. C: $75.71, \mathrm{H}: 6.11, \mathrm{~N}: 6.79$ found $\mathrm{C}: 75.45, \mathrm{H}: 5.97$, $\mathrm{N}: 6.57$.

The enantiomers of $\mathbf{4 b}$ were separated via preparative chiral HPLC (Chiralpak AD-H, $n$ heptane:EtOH 70:3, rt, $11.0 \mathrm{ml} / \mathrm{min}, 295 \mathrm{~nm})$; (-)-enantiomer : $5.7 \mathrm{~min},[\alpha]_{\mathrm{D}}{ }^{20}=-9.4(\mathrm{c}=$ 1.00, $\left.\mathrm{CHCl}_{3}\right)$; (+)-enantiomer: $11.9 \mathrm{~min},[\alpha]_{\mathrm{D}}^{20}=+10.1\left(\mathrm{c}=1.01, \mathrm{CHCl}_{3}\right)$.

(The analytical and spectroscopic data of the enantiomers correspond to the racemate) 


\section{6-Brom-2- $N$-trifluororacetylaminopyridine}

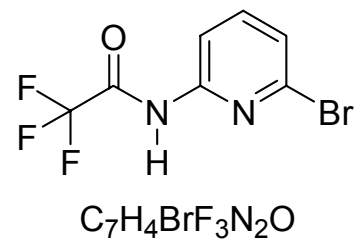

Mol. Wt.: 269.02

To a solution of $7.3 \mathrm{ml}$ trifluoroacetic acid anhydride (10.92 g, $52.0 \mathrm{mmol}, 3.0$ eq.) in $40 \mathrm{ml}$ $\mathrm{CH}_{2} \mathrm{Cl}_{2}$ was added slowly at $0^{\circ} \mathrm{C} 7.2 \mathrm{ml}$ triethylamine $(5.26 \mathrm{~g}, 52.0 \mathrm{mmol}, 3.0$ eq.). After 5 $\min 3.00 \mathrm{~g}$ 2-amino-6-bromopyridine (17.3 mmol, 1.0 eq.) was added and the solution was stirred at room temperature for 3 days. The conversion was monitored via TLC. After addition of $30 \mathrm{ml}$ sat. aqueous $\mathrm{NaHCO}_{3}$ solution, the aqueous layer was extracted with EtOAc $(4 \times$ $30 \mathrm{ml})$, the combined organic layers were dried $\left(\mathrm{MgSO}_{4}\right)$ and concentrated. The crude product was purified via flash chromatography (Cy:EtOAc 5:1) to yield $3.96 \mathrm{~g}$ of the title compound (14.7 $\mathrm{mmol}, 85 \%)$ as a yellow oil.

${ }^{1}$ H-NMR $\left(500 \mathrm{MHz}, \mathrm{CDCl}_{3}\right): \delta 8.61(\mathrm{br} \mathrm{s}, 1 \mathrm{H}), 8.15(\mathrm{~d}, J=8.2 \mathrm{~Hz}, 1 \mathrm{H}), 7.66$ (pt, $J=8.0 \mathrm{~Hz}$, 1H), $7.36(\mathrm{~d}, J=7.7 \mathrm{~Hz}, 1 \mathrm{H}) ;{ }^{13} \mathbf{C}-\mathbf{N M R}\left(125 \mathrm{MHz}, \mathrm{CDCl}_{3}\right): \delta 155.0\left(\mathrm{q}, J_{\mathrm{C}, \mathrm{F}}=38.8 \mathrm{~Hz}\right)$, 148.9, 141.1, 139.9, 125.9, 115.4 (q, $J_{\mathrm{C}, \mathrm{F}}=288.6 \mathrm{~Hz}$ ), 111.9; CHN: calcd. C: 31.25, H: 1.50, $\mathrm{N}: 10.41$ found $\mathrm{C}: 31.40, \mathrm{H}: 1.20, \mathrm{~N}: 10.36$. 
(rac)-6-(o-Tolylphenylphosphino)-2-aminopyridine

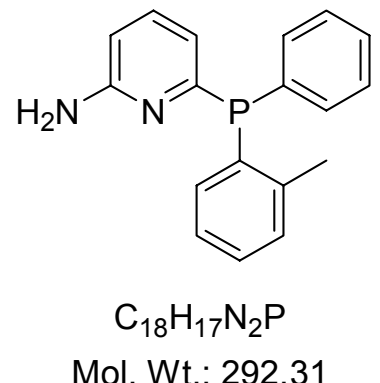

To a solution of $1.88 \mathrm{~g}$ 6-bromo-2- $N$-trifluoroacetylaminopyridine (7.0 mmol, 0.95 eq.) in $40 \mathrm{ml}$ THF was added at $-100^{\circ} \mathrm{C} 8.8 \mathrm{ml} n$-butyl-lithium $(1.60 \mathrm{M}$ in hexane, $14.0 \mathrm{mmol}$, 1.90 eq.) during $20 \mathrm{~min}$. The mixture was stirred for $90 \mathrm{~min}$, then a solution of $1.73 \mathrm{~g} o$ tolylphenylchlorophosphine (7.4 mmol, 1.0 eq.) in $10 \mathrm{ml}$ THF was slowly added. After stirring $30 \mathrm{~min}$ at $-100^{\circ} \mathrm{C}$, the yellow solution was allowed to warm to room temperature and stirred $12 \mathrm{~h}$ at this temperature. The solvent was removed under reduced pressure and the residue was filtrated through a plug of silica to remove salts. The filtrate was concentrated in vacuo and purified via flash chromatography (Cy:EtOAc 10:1) to furnish $1.12 \mathrm{~g}$ of the protected title compound ( $4.7 \mathrm{mmol}, 41 \%$ ) as a white foam.

$500 \mathrm{mg}(1.29 \mathrm{mmol})$ were directly deprotected by stirring in $10 \mathrm{ml} \mathrm{MeOH}$ with $1.78 \mathrm{~g} \mathrm{~K}_{2} \mathrm{CO}_{3}$ $(12.29 \mathrm{mmol})$ at $60^{\circ} \mathrm{C}$ for $4 \mathrm{~h}$. After addition of $5 \mathrm{ml}$ sat. aqueous $\mathrm{NaHCO}_{3}$ solution, the aqueous layer was extracted with EtOAc $(4 \times 20 \mathrm{ml})$, the combined organic layers were dried $\left(\mathrm{MgSO}_{4}\right)$ and concentrated. The crude product was purified via flash chromatography (Cy:EtOAc $1: 1)$ to yield $0.33 \mathrm{~g}$ of the title compound $(1.14 \mathrm{mmol}, 88 \%)$ as a white solid.

${ }^{1} \mathbf{H}-\mathbf{N M R}\left(400 \mathrm{MHz}, \mathrm{CDCl}_{3}\right): \delta$ 7.26-7.23 (m, 5H), 7.20-7.11 (m, 3H), 7.01 (pt, $J=7.2 \mathrm{~Hz}$, $1 \mathrm{H}), 6.78-6.75(\mathrm{~m}, 1 \mathrm{H}), 6.26(\mathrm{~d}, J=8.3 \mathrm{~Hz}, 1 \mathrm{H}), 6.21(\mathrm{~d}, J=7.3 \mathrm{~Hz}, 1 \mathrm{H}), 4.50(\mathrm{br} \mathrm{s}, 2 \mathrm{H})$, $2.35(\mathrm{~s}, 3 \mathrm{H}) ;{ }^{13} \mathrm{C}-\mathbf{N M R}\left(100 \mathrm{MHz}, \mathrm{CDCl}_{3}\right): \delta 159.9\left(\mathrm{~d}, J_{\mathrm{C}, \mathrm{P}}=9.2 \mathrm{~Hz}\right), 157.5\left(\mathrm{~d}, J_{\mathrm{C}, \mathrm{P}}=\right.$ $16.4 \mathrm{~Hz}), 141.7\left(\mathrm{~d}, J_{\mathrm{C}, \mathrm{P}}=25.4 \mathrm{~Hz}\right), 136.3\left(\mathrm{~d}, J_{\mathrm{C}, \mathrm{P}}=1.5 \mathrm{~Hz}\right), 134.5\left(\mathrm{~d}, J_{\mathrm{C}, \mathrm{P}}=10.6 \mathrm{~Hz}\right), 134.3$ $\left(\mathrm{d}, J_{\mathrm{C}, \mathrm{P}}=12.3 \mathrm{~Hz}\right), 133.4\left(\mathrm{~d}, J_{\mathrm{C}, \mathrm{P}}=19.8 \mathrm{~Hz}\right), 132.1,129.0\left(\mathrm{~d}, J_{\mathrm{C}, \mathrm{P}}=4.8 \mathrm{~Hz}\right), 127.9,127.8$, $127.5\left(\mathrm{~d}, J_{\mathrm{C}, \mathrm{P}}=7.3 \mathrm{~Hz}\right), 125.0\left(\mathrm{~d}, J_{\mathrm{C}, \mathrm{P}}=1.0 \mathrm{~Hz}\right), 117.6\left(\mathrm{~d}, J_{\mathrm{C}, \mathrm{P}}=11.1 \mathrm{~Hz}\right), 106.8,20.2\left(\mathrm{~d}, J_{\mathrm{C}, \mathrm{P}}\right.$ $=21.3 \mathrm{~Hz}) ;{ }^{31} \mathbf{P}-\mathbf{N M R}\left(121 \mathrm{MHz}, \mathrm{CDCl}_{3}\right): \delta-12.1$; CHN: calcd. C: 73.96, H: 5.86, N: 9.58 found $\mathrm{C}: 73.65, \mathrm{H}: 5.87, \mathrm{~N}: 9.50$.

The enantiomers were separated via preparative chiral HPLC (Chiralpak AD-H, nheptane:EtOH 95:5, rt, $11.0 \mathrm{ml} / \mathrm{min}, 282 \mathrm{~nm})$; (-)-enantiomer : $6.8 \mathrm{~min},[\alpha]_{\mathrm{D}}{ }^{21}=-17.3(\mathrm{c}=$ 1.00, $\left.\mathrm{CHCl}_{3}\right)$; (+)-enantiomer: $8.5 \mathrm{~min},[\alpha]_{\mathrm{D}}{ }^{21}=+17.1\left(\mathrm{c}=1.0, \mathrm{CHCl}_{3}\right)$.

(The analytical and spectroscopic data of the enantiomers correspond to the racemate) 
$(+)$

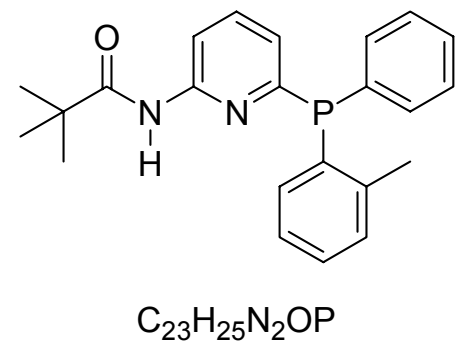

Mol. Wt.: 376.43

To a solution of $94 \mathrm{mg}(+)-6$-(o-tolylphenylphosphino)-2-aminopyridine (0.32 mmol, 1.0 eq.) in $25 \mathrm{ml} \mathrm{CH} \mathrm{Cl}_{2}$ was added consecutively at $0^{\circ} \mathrm{C} 85 \mu$ triethylamine $(62 \mathrm{mg}, 0.61 \mathrm{mmol}$, 1.9 eq.) and $59 \mu \mathrm{l}$ pivaloylchloride ( $58 \mathrm{mg}, 0.48 \mathrm{mmol}, 1.5$ eq.). After warming to room temperature the solution was stirred for $36 \mathrm{~h}$. The solvent was removed in vacuo and the residue was purified via flash chromatography (Cy:EtOAc 5:1) to yield $108 \mathrm{mg}$ of the title compound $(0.29 \mathrm{mmol}, 89 \%)$ as a white foam.

${ }^{1}$ H-NMR (500 MHz, $\left.\mathrm{C}_{6} \mathrm{D}_{6}\right): \delta 8.52(\mathrm{~d}, J=8.5 \mathrm{~Hz}, 1 \mathrm{H}), 7.89$ (br s, 1H) 7.44-7.41 (m, 2H), 7.10-6.99 (m, 7H), 6.94 (dd $J=7.4,7.4 \mathrm{~Hz}, 1 \mathrm{H}), 6.70$ (d, $J=7.7 \mathrm{~Hz}, 1 \mathrm{H}), 2.44(\mathrm{~s}, 3 \mathrm{H}), 0.85$ $(\mathrm{s}, 9 \mathrm{H}) ;{ }^{13} \mathbf{C}-\mathbf{N M R}\left(125 \mathrm{MHz}, \mathrm{C}_{6} \mathrm{D}_{6}\right): \delta 176.5,162.0\left(\mathrm{~d}, J_{\mathrm{C}, \mathrm{P}}=5.5 \mathrm{~Hz}\right), 152.8\left(\mathrm{~d}, J_{\mathrm{C}, \mathrm{P}}=14.8\right.$ $\mathrm{Hz}), 143.1\left(\mathrm{~d}, J_{\mathrm{C}, \mathrm{P}}=26.0 \mathrm{~Hz}\right), 138.2\left(\mathrm{~d}, J_{\mathrm{C}, \mathrm{P}}=1.2 \mathrm{~Hz}\right), 136.1\left(\mathrm{~d}, J_{\mathrm{C}, \mathrm{P}}=11.5 \mathrm{~Hz}\right), 135.8\left(\mathrm{~d}, J_{\mathrm{C}, \mathrm{P}}\right.$ $=12.7 \mathrm{~Hz}), 134.9\left(\mathrm{~d}, J_{\mathrm{C}, \mathrm{P}}=20.6 \mathrm{~Hz}\right), 133.7,130.5\left(\mathrm{~d}, J_{\mathrm{C}, \mathrm{P}}=4.9 \mathrm{~Hz}\right), 129.2\left(\mathrm{~d}, J_{\mathrm{C}, \mathrm{P}}=3.0 \mathrm{~Hz}\right)$, $129.0\left(\mathrm{~d}, J_{\mathrm{C}, \mathrm{P}}=7.3 \mathrm{~Hz}\right), 126.6,124.0\left(\mathrm{~d}, J_{\mathrm{C}, \mathrm{P}}=11.5 \mathrm{~Hz}\right), 112.8,39.5,27.0,21.3\left(\mathrm{~d}, J_{\mathrm{C}, \mathrm{P}}=11.8\right.$ Hz); ${ }^{31}$ P-NMR (121 MHz, $\left.\mathrm{C}_{6} \mathrm{D}_{6}\right): \delta-11.7$; HRMS: calcd. 376.1704 found $376.1693 ;[\alpha]_{\mathbf{D}}{ }^{\mathbf{2 0}}=$ $+4.0\left(\mathrm{c}=1.00, \mathrm{CHCl}_{3}\right)$.

The enantiomer (-)-4c was synthesized as described above starting from (-)-6-(otolylphenylphosphino)-2-aminopyridine.

(The analytical and spectroscopic data correspond to the $(+)$-enantiomer)

$[\alpha]_{\mathbf{D}}{ }^{20}=-3.9\left(\mathrm{c}=1.05, \mathrm{CHCl}_{3}\right)$. 


\section{6-(Bisdiethylaminophosphino)-2-pivaloylaminopyridine}

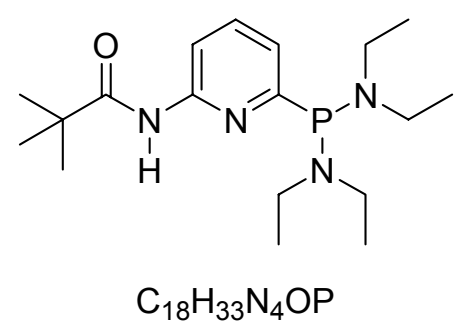

Mol. Wt.: 352.45

To a solution of 2-bromo-6- $N$-pivaloylaminopyridine (7.7 mmol, 1.0 eq.) in $75 \mathrm{ml}$ THF was added slowly at $-100^{\circ} \mathrm{C} 10.0 \mathrm{ml} n$-butyl-lithium (1.54 $\mathrm{M}$ in hexane, $15.4 \mathrm{mmol}, 2.0$ eq.). The mixture was stirred for $90 \mathrm{~min}$ at $-100^{\circ} \mathrm{C}$, then $1.62 \mathrm{~g}$ bisdiethylamino-chloro-phosphine (7.7 mmol, 1.0 eq.) was added in one portion. The solution was allowed to warm to room temperature overnight. The solvent was removed under reduced pressure, then $40 \mathrm{ml} \mathrm{Et}_{2} \mathrm{O}$ and $140 \mu \mathrm{l}$ degassed water (140 mg, $7.7 \mathrm{mmol}, 1.0$ eq.) were added successively and the resulting suspension was filtered over Celite ${ }^{\circledR}$ and $\mathrm{MgSO}_{4}$ under an argon atmosphere. After removal of the solvent in vacuo, the crude product was purified by bulb-to-bulb distillation $\left(60^{\circ} \mathrm{C} / 1 \cdot 10^{-3}\right.$ mbar to remove impurity, $\left.205^{\circ} \mathrm{C} / 1 \cdot 10^{-3} \mathrm{mbar}\right)$ to yield $1.41 \mathrm{~g}$ of the title compound (4.0 mmol, 52\%) as a yellow oil.

${ }^{1}$ H-NMR $\left(300 \mathrm{MHz}, \mathrm{C}_{6} \mathrm{D}_{6}\right): \delta 8.48(\mathrm{dd}, J=5.0,4.0 \mathrm{~Hz}, 1 \mathrm{H}), 8.01$ (br s, $\left.1 \mathrm{H}\right), 7.25$ (d, $J=$ $3.1 \mathrm{~Hz}, 2 \mathrm{H}), 3.07(\mathrm{~m}, 8 \mathrm{H}), 1.10(\mathrm{t}, J=7.0 \mathrm{~Hz}, 12 \mathrm{H}), 1.05(\mathrm{~s}, 9 \mathrm{H}) ;{ }^{13} \mathbf{C}-\mathbf{N M R}(100 \mathrm{MHz}$, $\left.\mathrm{C}_{6} \mathrm{D}_{6}\right): \delta 176.1,164.8\left(\mathrm{~d}, J_{\mathrm{C}, \mathrm{P}}=13.1 \mathrm{~Hz}\right), 152.4\left(\mathrm{~d}, J_{\mathrm{C}, \mathrm{P}}=7.3 \mathrm{~Hz}\right), 137.6,122.8\left(\mathrm{~d}, J_{\mathrm{C}, \mathrm{P}}=\right.$ $21.8 \mathrm{~Hz}), 111.6\left(\mathrm{~d}, J_{\mathrm{C}, \mathrm{P}}=2.9 \mathrm{~Hz}\right), 43.9\left(\mathrm{~d}, J_{\mathrm{C}, \mathrm{P}}=17.4 \mathrm{~Hz}\right), 39.6,27.3,14.9\left(\mathrm{~d}, J_{\mathrm{C}, \mathrm{P}}=2.9 \mathrm{~Hz}\right)$;

${ }^{31}$ P-NMR (121 MHz, $\mathrm{C}_{6} \mathrm{D}_{6}$ ): $\delta$ 94.3; CHN calcd. C: 61.34, H: 9.44, N: 15.90 found C: 61.30, H: 9.21, N: 15.57 . 


\section{General procedure: Synthesis of aminopyridinyl phosphonites}

6-(Bisdiethylaminophosphino)-2-pivaloylaminopyridine $(0.2-0.6 \mathrm{mmol})$ was dissolved in toluene $(44 \mathrm{ml} / \mathrm{mmol})$ and the enantiomerically pure BINOL-derivative (1.0 eq.) was added. The mixture was heated to $96^{\circ} \mathrm{C}$ in a closed Schlenk tube. Diethylamine formed during the reaction was removed several times under vacuum. Conversion was monitored by ${ }^{31} \mathrm{P}-\mathrm{NMR}$. The solvent was removed under reduced pressure and the residue taken up in pentane twice to give the title compound as a yellowish foam in quantitative yield.

\section{Analytical data for $(S)-5 a:$}

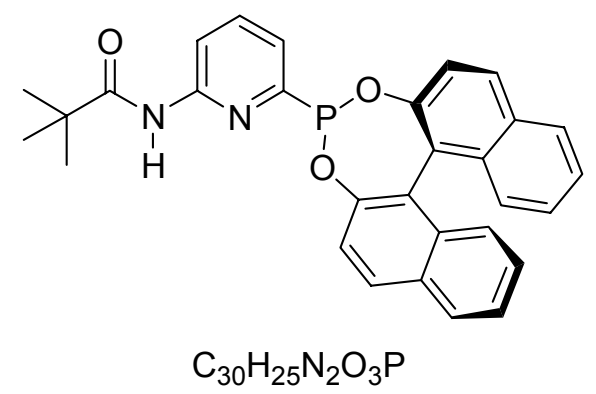

Mol. Wt.: 492.50

${ }^{1}$ H-NMR $\left(300 \mathrm{MHz}, \mathrm{C}_{6} \mathrm{D}_{6}\right): \delta 8.56(\mathrm{~d}, J=8.5 \mathrm{~Hz}, 1 \mathrm{H}), 8.10(\mathrm{br} \mathrm{s}, 1 \mathrm{H}), 7.65(\mathrm{~d}, J=8.6 \mathrm{~Hz}$, $1 \mathrm{H}), 7.61(\mathrm{~d}, J=8.8 \mathrm{~Hz}, 1 \mathrm{H}), 7.55-7.42(\mathrm{~m}, 4 \mathrm{H}), 7.30(\mathrm{~d}, J=8.7 \mathrm{~Hz}, 1 \mathrm{H}), 7.13-6.93(\mathrm{~m}, 5 \mathrm{H})$, $6.88(\mathrm{~d}, J=7.2 \mathrm{~Hz}, 1 \mathrm{H}), 6.77$ (dd, $J=8.1,7.6 \mathrm{~Hz}, 1 \mathrm{H}), 0.99(\mathrm{~s}, 9 \mathrm{H}) ;{ }^{13} \mathbf{C}-\mathbf{N M R}(100 \mathrm{MHz}$, $\left.\mathrm{C}_{6} \mathrm{D}_{6}\right): \delta 175.2,159.5\left(\mathrm{~d}, J_{\mathrm{C}, \mathrm{P}}=20.8 \mathrm{~Hz}\right), 152.2,151.4\left(\mathrm{~d}, J_{\mathrm{C}, \mathrm{P}}=16.2 \mathrm{~Hz}\right), 148.7\left(\mathrm{~d}, J_{\mathrm{C}, \mathrm{P}}=\right.$ $2.9 \mathrm{~Hz}), 148.2\left(\mathrm{~d}, J_{\mathrm{C}, \mathrm{P}}=6.5 \mathrm{~Hz}\right), 136.6,133.1,132.8\left(\mathrm{~d}, J_{\mathrm{C}, \mathrm{P}}=1.2 \mathrm{~Hz}\right), 132.2\left(\mathrm{~d}, J_{\mathrm{C}, \mathrm{P}}=\right.$ $1.4 \mathrm{~Hz}), 129.9,129.5,128.4\left(\mathrm{~d}, J_{\mathrm{C}, \mathrm{P}}=15.0 \mathrm{~Hz}\right), 127.4,127.3,125.9\left(\mathrm{~d}, J_{\mathrm{C}, \mathrm{P}}=9.2 \mathrm{~Hz}\right), 125.3$ $\left(\mathrm{d}, J_{\mathrm{C}, \mathrm{P}}=13.8 \mathrm{~Hz}\right), 124.3,124.0,122.9\left(\mathrm{~d}, J_{\mathrm{C}, \mathrm{P}}=2.9 \mathrm{~Hz}\right), 122.4,120.3\left(\mathrm{~d}, J_{\mathrm{C}, \mathrm{P}}=1.2 \mathrm{~Hz}\right)$, $119.4\left(\mathrm{~d}, J_{\mathrm{C}, \mathrm{P}}=9.9 \mathrm{~Hz}\right), 117.2,114.3,111.8,38.3,25.8 ;{ }^{31} \mathbf{P}-\mathbf{N M R}\left(121 \mathrm{MHz}, \mathrm{C}_{6} \mathrm{D}_{6}\right): \delta 167.6$; HRMS: calcd. 491.1535 found $491.1525 ;[\alpha]_{\mathbf{D}}{ }^{21}=-30.0\left(\mathrm{c}=0.57, \mathrm{CHCl}_{3}\right)$. 


\section{Analytical data for $(S)-5 b$ :}

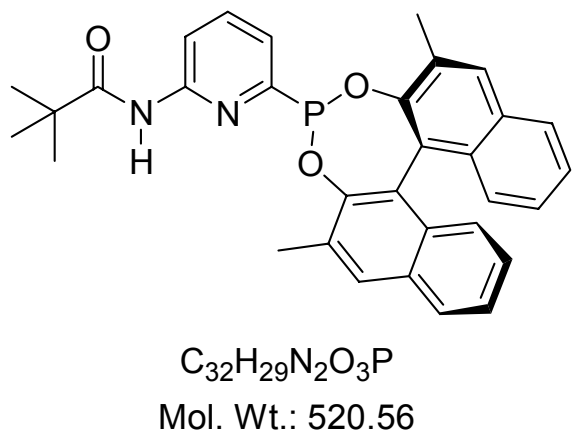

${ }^{1}$ H-NMR (400 MHz, $\left.\mathrm{C}_{6} \mathrm{D}_{6}\right): \delta 8.55(\mathrm{~d}, J=8.3 \mathrm{~Hz}, 1 \mathrm{H}), 8.04$ (br s, $\left.1 \mathrm{H}\right), 7.67(\mathrm{~d}, J=8.2 \mathrm{~Hz}$, 1H), 7.58 (dd, $J=9.4,8.2 \mathrm{~Hz}, 2 \mathrm{H}), 7.55$ (s, 1H), 7.45 (d, $J=8.5 \mathrm{~Hz}, 1 \mathrm{H}), 7.31(\mathrm{~s}, 1 \mathrm{H}), 7.22-$ $7.18(\mathrm{~m}, 2 \mathrm{H}), 7.00-6.93(\mathrm{~m}, 2 \mathrm{H}), 6.84(\mathrm{dd}, J=7.3,0.8 \mathrm{~Hz}, 1 \mathrm{H}), 6.74(\mathrm{dd}, J=8.0,7.7 \mathrm{~Hz}, 1 \mathrm{H})$, $2.49(\mathrm{~s}, 3 \mathrm{H}), 1.87(\mathrm{~s}, 3 \mathrm{H}), 0.98(\mathrm{~s}, 9 \mathrm{H}) ;{ }^{13} \mathrm{C}-\mathbf{N M R}\left(100 \mathrm{MHz}, \mathrm{C}_{6} \mathrm{D}_{6}\right): \delta 176.5,161.0\left(\mathrm{~d}, J_{\mathrm{C}, \mathrm{P}}=\right.$ $20.8 \mathrm{~Hz}), 152.6\left(\mathrm{~d}, J_{\mathrm{C}, \mathrm{P}}=17.4 \mathrm{~Hz}\right), 149.0\left(\mathrm{~d}, J_{\mathrm{C}, \mathrm{P}}=3.4 \mathrm{~Hz}\right), 148.6\left(\mathrm{~d}, J_{\mathrm{C}, \mathrm{P}}=6.8 \mathrm{~Hz}\right), 138.0$, $132.5\left(\mathrm{~d}, J_{\mathrm{C}, \mathrm{P}}=1.7 \mathrm{~Hz}\right), 132.2,131.6,130.9,130.5,130.1\left(\mathrm{~d}, J_{\mathrm{C}, \mathrm{P}}=1.5 \mathrm{~Hz}\right), 129.7\left(\mathrm{~d}, J_{\mathrm{C}, \mathrm{P}}=\right.$ $1.0 \mathrm{~Hz}), 127.4,127.2,125.8,125.7,125.7,125.5\left(\mathrm{~d}, J_{\mathrm{C}, \mathrm{P}}=6.0 \mathrm{~Hz}\right), 125.3,125.2,124.3(\mathrm{~d}$, $\left.J_{\mathrm{C}, \mathrm{P}}=2.9 \mathrm{~Hz}\right), 120.7\left(\mathrm{~d}, J_{\mathrm{C}, \mathrm{P}}=10.4 \mathrm{~Hz}\right), 115.9,39.6,27.1,17.2\left(\mathrm{~d}, J_{\mathrm{C}, \mathrm{P}}=1.9 \mathrm{~Hz}\right), 16.9 ;{ }^{31} \mathbf{P}-$ NMR (121 MHz, $\mathrm{C}_{6} \mathrm{D}_{6}$ ): $\delta$ 163.4; HRMS: calcd. 520.1908 found 520.1916; $[\alpha]_{\mathbf{D}}{ }^{21}=+22.5$ (c $\left.=0.44, \mathrm{CHCl}_{3}\right)$. 


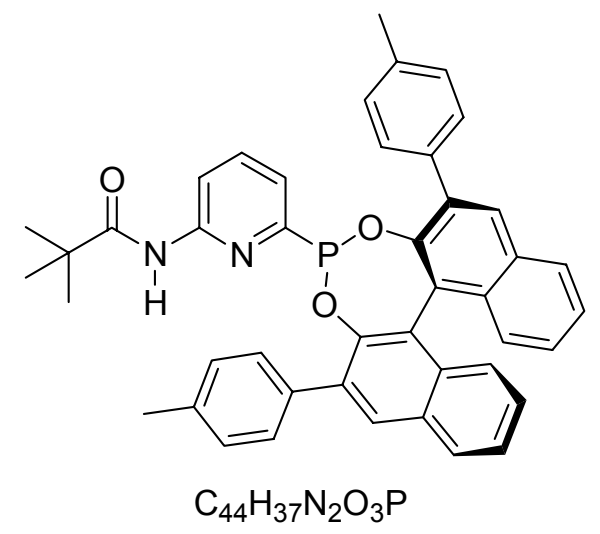

Mol. Wt.: 672.75

${ }^{1}$ H-NMR (400 MHz, $\left.\mathrm{C}_{6} \mathrm{D}_{6}\right): \delta 8.35$ (ddd, $\left.J=8.1,1.5,1.3 \mathrm{~Hz}, 1 \mathrm{H}\right), 7.98$ (s, 1H), 7.76 (d, $J=$ $8.1 \mathrm{~Hz}, 1 \mathrm{H}), 7.68(\mathrm{~s}, 1 \mathrm{H}), 7.69-7.66(\mathrm{~m}, 4 \mathrm{H}), 7.59$ (d, $J=8.3 \mathrm{~Hz}, 1 \mathrm{H}), 7.47$ (br s, 1H), 7.25 (ddd, $J=7.6,1.3,1.0 \mathrm{~Hz}, 1 \mathrm{H}), 7.20$ (ddd, $J=7.3,1.5,1.0 \mathrm{~Hz}, 1 \mathrm{H}), 7.05(\mathrm{~m}, 2 \mathrm{H}), 6.99$ (m, $4 \mathrm{H}), 6.88(\mathrm{~d}, J=8.1 \mathrm{~Hz}, 2 \mathrm{H}), 6.70(\mathrm{dd}, J=7.3,1.5 \mathrm{~Hz}, 1 \mathrm{H}), 6.67(\mathrm{dd}, J=8.1,7.3 \mathrm{~Hz}, 1 \mathrm{H})$, $2.14(\mathrm{~s}, 3 \mathrm{H}), 2.05(\mathrm{~s}, 3 \mathrm{H}), 1.00(\mathrm{~s}, 9 \mathrm{H}) ;{ }^{13} \mathrm{C}-\mathbf{N M R}\left(100 \mathrm{MHz}, \mathrm{C}_{6} \mathrm{D}_{6}\right): \delta 175.9,159.5\left(\mathrm{~d}, J_{\mathrm{C}, \mathrm{P}}=\right.$ $19.3 \mathrm{~Hz}), 152.0\left(\mathrm{~d}, J_{\mathrm{C}, \mathrm{P}}=17.4 \mathrm{~Hz}\right), 147.5\left(\mathrm{~d}, J_{\mathrm{C}, \mathrm{P}}=2.9 \mathrm{~Hz}\right), 146.9\left(\mathrm{~d}, J_{\mathrm{C}, \mathrm{P}}=5.8 \mathrm{~Hz}\right), 137.0(\mathrm{~d}$, $\left.J_{\mathrm{C}, \mathrm{P}}=20.3 \mathrm{~Hz}\right), 135.9,135.6,135.1\left(\mathrm{~d}, J_{\mathrm{C}, \mathrm{P}}=1.9 \mathrm{~Hz}\right), 135.0,134.8,133.0\left(\mathrm{~d}, J_{\mathrm{C}, \mathrm{P}}=1.9 \mathrm{~Hz}\right)$, $132.5,131.9,131.4,131.2,130.0\left(\mathrm{~d}, J_{\mathrm{C}, \mathrm{P}}=1.9 \mathrm{~Hz}\right), 129.8,129.5,129.0,128.6,128.5,128.4$, 127.1, 127.1, 126.4, 126.3, 126.3, 125.4, 125.3, 120.1 (d, $\left.J_{\mathrm{C}, \mathrm{P}}=10.6 \mathrm{~Hz}\right), 115.7,39.3,27.1$, 21.2, 20.9; ${ }^{31}$ P-NMR (121 MHz, $\mathrm{C}_{6} \mathrm{D}_{6}$ ): $\delta$ 164.9; HRMS: calcd. 672.2521 found 672.2542; $[\alpha]_{\mathbf{D}}{ }^{20}=+33.8\left(\mathrm{c}=0.76, \mathrm{CHCl}_{3}\right)$. 


\section{3-Bromo-1-(2-(trimethylsilyl)-ethoxy)isoquinoline}

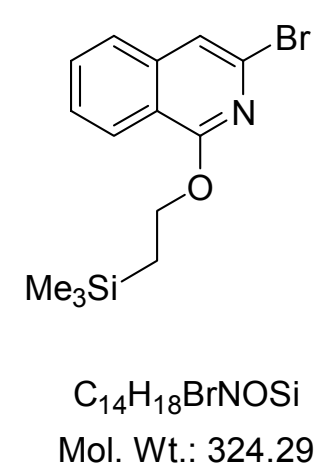

To a suspension of $2.60 \mathrm{~g}$ potassium-tert-butylate $(23.1 \mathrm{mmol}, 1.2$ eq.) in $80 \mathrm{ml}$ THF was added at $0^{\circ} \mathrm{C} 2.50 \mathrm{~g} \mathrm{2-(trimethylsilyl)ethanol} \mathrm{(21.2} \mathrm{mmol,} 1.1$ eq.) and the mixture was stirred at this temperature for $30 \mathrm{~min}$. Then a solution of $5.53 \mathrm{~g}$ 1,3-dibromoisoquinoline (19.3 mmol, 1.0 eq.) in $30 \mathrm{ml}$ THF was added slowly and the resulting suspension was stirred at room temperature for another $90 \mathrm{~min}$. The reaction mixture was quenched by addition of $100 \mathrm{ml} \mathrm{sat}$. aqueous $\mathrm{NH}_{4} \mathrm{Cl}$ solution, the aqueous layer was extracted with EtOAc $(3 \times 100 \mathrm{ml})$ and the combined organic layers were dried $\left(\mathrm{MgSO}_{4}\right)$ and concentrated under reduced pressure. The crude product was purified by flash chromatography on silica gel (Cy:EtOAc 95:5) to yield $6.00 \mathrm{~g}$ of the title compound ( $18.5 \mathrm{mmol}, 96 \%)$ as a colorless liquid.

${ }^{1} \mathbf{H}-\mathbf{N M R}\left(500 \mathrm{MHz}, \mathrm{CDCl}_{3}\right): \delta 8.19(\mathrm{~d}, J=8.3 \mathrm{~Hz}, 1 \mathrm{H}), 7.65-7.60(\mathrm{~m}, 2 \mathrm{H}), 7.51$ (ddd, $J=$ 7.4, 7.2, $1.9 \mathrm{~Hz}, 1 \mathrm{H}), 7.40(\mathrm{~s}, 1 \mathrm{H}), 4.63(\mathrm{~m}, 2 \mathrm{H}), 1.24(\mathrm{~m}, 2 \mathrm{H}), 0.13(\mathrm{~s}, 9 \mathrm{H}) ;{ }^{13} \mathrm{C}-\mathbf{N M R}$ $\left(125 \mathrm{MHz}, \mathrm{CDCl}_{3}\right): \delta 160.2,139.5,132.5,131.2,126.7,125.3,124.6,118.6,116.9,65.3$, 17.5, -1.2, CHN calcd. C: 51.85, H: 5.59, N: 4.32 found C: 51.71, H: 5.55, N: 4.26. 


\section{3-(Bisdiethylaminophosphino)isoquinolin-1(2H)-one}

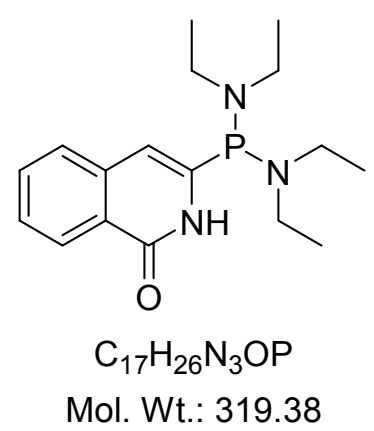

To a solution of $2.00 \mathrm{~g}$ 3-bromo-1-(2-(trimethylsilyl)-ethoxy)isoquinoline (6.2 mmol, 1.0 eq.) in $35 \mathrm{ml}$ THF was added slowly at $-100^{\circ} \mathrm{C} 4.7 \mathrm{ml} n$-butyl-lithium $(1.3 \mathrm{M}$ in hexane, $6.2 \mathrm{mmol}$, 1.0 eq.). The mixture was stirred for $20 \mathrm{~min}$ at this temperature, then $1.30 \mathrm{~g}$ bisdiethylamino-chloro-phosphine $(6.2 \mathrm{mmol}, 1.0$ eq.) was added in one portion. The solution was allowed to warm to room temperature and stirring was continued for another $20 \mathrm{~min}$. The reaction mixture was quenched by addition of $200 \mu \mathrm{l}$ degassed water and then concentrated in vacuo. The residue was transferred to a separating funnel with degassed water and $\mathrm{CH}_{2} \mathrm{Cl}_{2}$. The aqueous layer was separated and extracted with $\mathrm{CH}_{2} \mathrm{Cl}_{2}(2 \times 50 \mathrm{ml})$. The combined organic layers were dried $\left(\mathrm{MgSO}_{4}\right)$ and concentrated under reduced pressure to yield $2.58 \mathrm{~g}$ of crude protected 3-(bis(diethylamino)phosphino)isoquinoline $(6.15 \mathrm{mmol}$, quant.) as a yellow oil, which was immediately deprotected in the next step.

To a solution of $1.45 \mathrm{~g}$ protected 3-(bis(diethylamino)phosphino)isoquinoline (3.5 mmol, 1.0 eq.) in $15 \mathrm{ml}$ THF was added $3.8 \mathrm{ml}$ TBAF ( $1 \mathrm{M}$ in THF, $3.8 \mathrm{mmol}, 1.1$ eq.) and the mixture was stirred at room temperature for $3 \mathrm{~h}$. The reaction was quenched by addition of $15 \mathrm{ml}$ degassed water and $50 \mathrm{ml} \mathrm{Et}_{2} \mathrm{O}$. The organic layer was separated and washed with degassed water $(4 \times 15 \mathrm{ml})$ and dried $\left(\mathrm{MgSO}_{4}\right)$. The crude solid was washed with $\mathrm{Et}_{2} \mathrm{O}: \mathrm{PE}$ (30-50) 1:4 several times to yield $0.60 \mathrm{~g}$ of the title compound (1.9 mmol, 54\%) as a cream coloured solid.

${ }^{1}$ H-NMR (300 MHz, $\left.\mathrm{C}_{6} \mathrm{D}_{6}\right): \delta 8.91$ (br s, $\left.1 \mathrm{H}\right), 8.78(\mathrm{~d}, J=7.9 \mathrm{~Hz}, 1 \mathrm{H}), 7.27-7.11(\mathrm{~m}, 3 \mathrm{H})$, $6.50(\mathrm{~s}, 1 \mathrm{H}), 2.92-2.80(\mathrm{~m}, 8 \mathrm{H}), 0.91(\mathrm{pt}, J=7.0 \mathrm{~Hz}, 12 \mathrm{H}) ;{ }^{13} \mathbf{C}-\mathbf{N M R}\left(100 \mathrm{MHz}, \mathrm{C}_{6} \mathrm{D}_{6}\right): \delta$ $162.9,142.1\left(\mathrm{~d}, J_{\mathrm{C}, \mathrm{P}}=4.4 \mathrm{~Hz}\right), 138.5\left(\mathrm{~d}, J_{\mathrm{C}, \mathrm{P}}=2.9 \mathrm{~Hz}\right), 132.4,128.2,127.9,126.7,126.2$, $109.1\left(\mathrm{~d}, J_{\mathrm{C}, \mathrm{P}}=16.0 \mathrm{~Hz}\right), 43.4\left(\mathrm{~d}, J_{\mathrm{C}, \mathrm{P}}=17.4 \mathrm{~Hz}\right), 14.6\left(\mathrm{~d}, J_{\mathrm{C}, \mathrm{P}}=4.4 \mathrm{~Hz}\right) ;{ }^{31} \mathbf{P}-\mathbf{N M R}$ (121 MHz, $\left.\mathrm{C}_{6} \mathrm{D}_{6}\right): \delta$ 87.8; HRMS: calcd. 319.1816 found 319.1814. 


\section{$(S)$-and (R)-6a:}

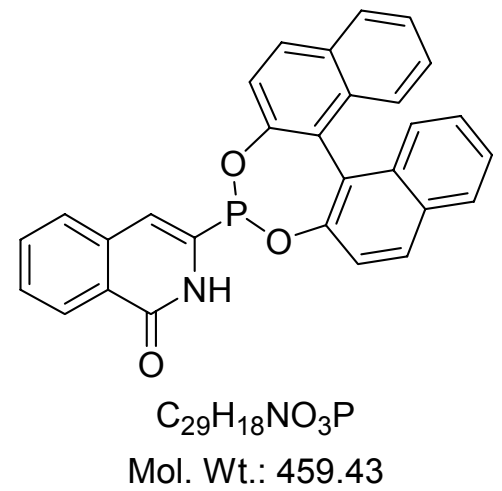

To a solution of $0.11 \mathrm{~g}$ 3-(bisdiethylaminophosphino)isoquinolin-1(2H)-one (0.34 mmol, 1.0 eq.) in $12 \mathrm{ml}$ toluene was added $0.10 \mathrm{~g}$ binaphtol ( $0.35 \mathrm{mmol}, 1.0$ eq. $)$. The mixture was heated at reflux under a stream of dry argon until the volume of solvent had been reduced to $1 \mathrm{ml}$. Toluene was added and the mixture heated to reflux for several hours. Conversion was monitored by ${ }^{31} \mathrm{P}-\mathrm{NMR}$. The solvent was removed under reduced pressure, the residue purified by adding $10 \mathrm{ml} \mathrm{Et}_{2} \mathrm{O}$ and filtring off the insolubles. The filtrate was concentrated in vacuo to yield $0.13 \mathrm{~g}$ of the title compound $\mathbf{6 a}(0.27 \mathrm{mmol}, 80 \%)$ as a cream coloured solid.

${ }^{1}$ H-NMR $\left(400 \mathrm{MHz}, \mathrm{C}_{6} \mathrm{D}_{6}\right): \delta 8.90$ (br s, $\left.1 \mathrm{H}\right), 8.65$ (d, $\left.J=8.0 \mathrm{~Hz}, 1 \mathrm{H}\right), 7.64$ (dd, $J=8.8$, $8.1 \mathrm{~Hz}, 2 \mathrm{H}), 7.49$ (d, $J=8.1 \mathrm{~Hz}, 1 \mathrm{H}), 7.40$ (m, 3H), 7.34 (d, $J=8.9 \mathrm{~Hz}, 1 \mathrm{H}), 7.05-6.89$ (m, $7 \mathrm{H}), 6.84(\mathrm{ddd}, J=7.7,1.0 \mathrm{~Hz}, 1 \mathrm{H}), 6.62(\mathrm{~d}, J=10.1 \mathrm{~Hz}, 1 \mathrm{H}) ;{ }^{13} \mathbf{C}-\mathbf{N M R}\left(100 \mathrm{MHz}, \mathrm{C}_{6} \mathrm{D}_{6}\right)$ : $\delta 161.3,153.5,149.2\left(\mathrm{~d}, J_{\mathrm{C}, \mathrm{P}}=10.2 \mathrm{~Hz}\right), 149.2\left(\mathrm{~d}, J_{\mathrm{C}, \mathrm{P}}=2.0 \mathrm{~Hz}\right), 140.3\left(\mathrm{~d}, J_{\mathrm{C}, \mathrm{P}}=52.1 \mathrm{~Hz}\right)$, $136.5\left(\mathrm{~d}, J_{\mathrm{C}, \mathrm{P}}=11.8 \mathrm{~Hz}\right), 134.3,133.4\left(\mathrm{~d}, J_{\mathrm{C}, \mathrm{P}}=1.7 \mathrm{~Hz}\right), 132.7\left(\mathrm{~d}, J_{\mathrm{C}, \mathrm{P}}=1.0 \mathrm{~Hz}\right), 132.5,132.2$ $\left(\mathrm{d}, J_{\mathrm{C}, \mathrm{P}}=1.0 \mathrm{~Hz}\right), 131.3,131.2\left(\mathrm{~d}, J_{\mathrm{C}, \mathrm{P}}=6.5 \mathrm{~Hz}\right), 127.6,127.3,127.1,127.0,126.9,125.5(\mathrm{~d}$, $\left.J_{\mathrm{C}, \mathrm{P}}=10.9 \mathrm{~Hz}\right), 124.8,124.0,123.3\left(\mathrm{~d}, J_{\mathrm{C}, \mathrm{P}}=2.7 \mathrm{~Hz}\right), 121.3\left(\mathrm{~d}, J_{\mathrm{C}, \mathrm{P}}=1.5 \mathrm{~Hz}\right), 121.0,118.3$, $114.3\left(\mathrm{~d}, J_{\mathrm{C}, \mathrm{P}}=42.0 \mathrm{~Hz}\right), 112.0 ;{ }^{31} \mathbf{P}-\mathbf{N M R}\left(121 \mathrm{MHz}, \mathrm{C}_{6} \mathrm{D}_{6}\right): \delta$ 162.2; HRMS: calcd. 459.1023 found 459.1024; $(S)-6 \mathbf{a}:[\alpha]_{\mathbf{D}}{ }^{21}=+95.3\left(\mathrm{c}=0.60, \mathrm{CHCl}_{3}\right) ;(R)-\mathbf{6 a}:[\alpha]_{\mathbf{D}}{ }^{20}=-104.1$ $\left(\mathrm{c}=0.66, \mathrm{CHCl}_{3}\right)$. 
(S)-6b:

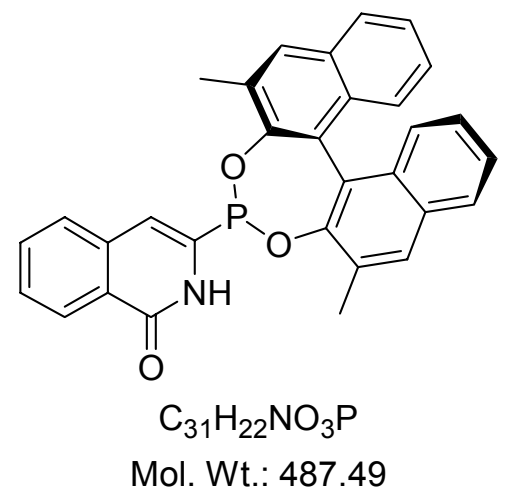

To a solution of $0.22 \mathrm{~g}$ 3-(bis(diethylamino)phosphino)isoquinolin-1(2H)-one (0.69 mmol, 1.00 eq.) in $6 \mathrm{ml} m$-xylene was added $0.16 \mathrm{~g}(S)$-3,3'-dimethyl-2,2'-dihydroxy-1,1'-binaphtyl ( $0.52 \mathrm{mmol}, 0.75$ eq.). The mixture was heated at reflux under a stream of dry argon until the volume of solvent had been reduced to $1 \mathrm{ml}$. Again $m$-xylene was added and the mixture heated to reflux for another 3-6 h. Conversion was monitored by ${ }^{31} \mathrm{P}-\mathrm{NMR}$. The solvent was removed under reduced pressure, the residue purified by adding $15 \mathrm{ml} \mathrm{Et}_{2} \mathrm{O}$ and filtring off the insolubles. The filtrate was concentrated in vacuo to furnish $0.24 \mathrm{~g}$ of the title compound $(S)-6 \mathbf{b}(0.48 \mathrm{mmol}, 92 \%)$ as a white solid.

${ }^{1}$ H-NMR $\left(400 \mathrm{MHz}, \mathrm{C}_{6} \mathrm{D}_{6}\right): \delta 8.51(\mathrm{~d}, J=7.8 \mathrm{~Hz}, 1 \mathrm{H}), 8.27(\mathrm{br} \mathrm{s}, 1 \mathrm{H}), 7.67(\mathrm{~d}, J=8.2 \mathrm{~Hz}$, 1H), 7.59 (d, $J=8.2 \mathrm{~Hz}, 1 \mathrm{H}), 7.55(\mathrm{~s}, 1 \mathrm{H}), 7.43$ (pt, $J=8.1 \mathrm{~Hz}, 2 \mathrm{H}), 7.36(\mathrm{~s}, 1 \mathrm{H}), 7.22$ (d, $J=$ $7.2 \mathrm{~Hz}, 1 \mathrm{H}), 7.13-6.85(\mathrm{~m}, 6 \mathrm{H}), 6.62(\mathrm{~d}, J=12.0 \mathrm{~Hz}, 1 \mathrm{H}), 2.44(\mathrm{~s}, 3 \mathrm{H}), 1.96(\mathrm{~s}, 3 \mathrm{H}) ;{ }^{13} \mathrm{C}-$ NMR (100 MHz, $\left.\mathrm{C}_{6} \mathrm{D}_{6}\right): \delta 160.4,152.4,148.3\left(\mathrm{~d}, J_{\mathrm{C}, \mathrm{P}}=5.8 \mathrm{~Hz}\right), 148.0\left(\mathrm{~d}, J_{\mathrm{C}, \mathrm{P}}=2.9 \mathrm{~Hz}\right)$, $140.5\left(\mathrm{~d}, J_{\mathrm{C}, \mathrm{P}}=52.2 \mathrm{~Hz}\right), 136.1\left(\mathrm{~d}, J_{\mathrm{C}, \mathrm{P}}=13.5 \mathrm{~Hz}\right), 132.7,132.2,132.1\left(\mathrm{~d}, J_{\mathrm{C}, \mathrm{P}}=1.9 \mathrm{~Hz}\right)$, $132.0\left(\mathrm{~d}, J_{\mathrm{C}, \mathrm{P}}=0.7 \mathrm{~Hz}\right), 131.5,130.9,130.7,130.7,129.8,129.6\left(\mathrm{~d}, J_{\mathrm{C}, \mathrm{P}}=1.9 \mathrm{~Hz}\right), 128.5$, 127.1, 127.0, $126.9\left(\mathrm{~d}, J_{\mathrm{C}, \mathrm{P}}=1.9 \mathrm{~Hz}\right), 126.5,125.9\left(\mathrm{~d}, J_{\mathrm{C}, \mathrm{P}}=12.1 \mathrm{~Hz}\right), 125.4\left(\mathrm{~d}, J_{\mathrm{C}, \mathrm{P}}=\right.$ $2.9 \mathrm{~Hz}), 124.3,123.9,114.6\left(\mathrm{~d}, J_{\mathrm{C}, \mathrm{P}}=46.4 \mathrm{~Hz}\right), 111.0,16.9,16.7 ;{ }^{31} \mathbf{P}-\mathbf{N M R}(121 \mathrm{MHz}$, $\left.\mathrm{C}_{6} \mathrm{D}_{6}\right): \delta 156.5$; HRMS: calcd. 487.1320 found $487.1337 ;[\alpha]_{\mathbf{D}}{ }^{21}=-175.7\left(\mathrm{c}=0.51, \mathrm{CHCl}_{3}\right)$. 


\section{General procedure for asymmetric hydrogenation}

$2.0 \mathrm{mg}\left[\mathrm{Rh}(\mathrm{COD})_{2}\right] \mathrm{BF}_{4}\left(5.0 \cdot 10^{-3} \mathrm{mmol}, 1.0 \mathrm{~mol} \%\right), \mathbf{L}_{1}\left(5.5-6.5 \cdot 10^{-3} \mathrm{mmol}, 1.1-1.3 \mathrm{~mol} \%\right)$ and $\mathbf{L}_{\mathbf{2}}\left(5.5-6.5 \cdot 10^{-3} \mathrm{mmol}, 1.1-1.3 \mathrm{~mol} \%\right)$ were dissolved in $5 \mathrm{ml}$ of solvent and stirred at room temperature for $30 \mathrm{~min}$. Subsequently the appropriate substrate $(0.5 \mathrm{mmol}, 1$ eq. $)$ was added in one portion.

For hydrogenation at atmospheric pressure, the reaction vessel was evacuated and refilled with hydrogen gas four times. Following this procedure, the yellow reaction mixture was vigorously stirred under an atmosphere of hydrogen (hydrogen balloon) at the indicated temperature.

For hydrogenations performed at higher pressure, the reaction mixture was transferred into a steel autoclave via syringe under an argon atmosphere. The reaction mixture was saturated with hydrogen gas by applying four cycles of careful evacuation and refilling with hydrogen gas followed by adjustment of the reaction pressure indicated.

Conversion was determined by ${ }^{1} \mathrm{H}-\mathrm{NMR}$ after $24 \mathrm{~h}$.

\section{Hydrogenation of acetamidoacrylate:}

Enantiomeric excess was determined by chiral GC analysis using a Hydrodex- $\beta$-TBDAc column, $25 \mathrm{~m} \times 0.25 \mathrm{~mm}, 120^{\circ} \mathrm{C}$

$t_{\mathrm{R}}[(R)$-enantiomer $]=14.0 \mathrm{~min}$.

$t_{\mathrm{R}}[(S)$-enantiomer $]=20.3 \mathrm{~min}$.

\section{Hydrogenation of methyl- $\alpha$-acetylamino cinnamate 7 :}

Enantiomeric excess was determined by chiral HPLC analysis using a Chiralpak-AD column, $25 \mathrm{~cm} \times 4.6 \mathrm{~mm}, n$-heptane:i-propanol 90:10, $254 \mathrm{~nm}$

$t_{\mathrm{R}}[(R)$-enantiomer $]=15.0 \mathrm{~min}$.

$t_{\mathrm{R}}[(S)$-enantiomer $]=19.5 \mathrm{~min}$.

\section{Hydrogenation of dimethylitaconate $\mathbf{8}$ :}

Enantiomeric excess was determined by chiral GC analysis using a G-TA, Trifluoroacetyl- $\gamma$ Cyclodextrin column, $30 \mathrm{~m} \times 0.5 \mathrm{~mm}, 75^{\circ} \mathrm{C}$

$t_{\mathrm{R}}[(S)$-enantiomer $]=32.2 \mathrm{~min}$.

$t_{\mathrm{R}}[(R)$-enantiomer $]=35.2 \mathrm{~min}$. 


\section{Heterodimeric platinum complexes}

\section{Platinum complex of $(R)-6 a$ and 6-DPPAP:}

To a solution of $14.5 \mathrm{mg}$ [cis- $\left.\mathrm{PtCl}_{2}(1,5-\mathrm{COD})\right]\left(4.01 \cdot 10^{-2} \mathrm{mmol}, 1.0\right.$ eq.) in $0.4 \mathrm{ml} \mathrm{CDCl}_{3}$ was added a solution of $18.4 \mathrm{mg}$ isoquinolone $(R)-6 \mathrm{a}\left(4.01 \cdot 10^{-2} \mathrm{mmol}, 1.0 \mathrm{eq}.\right)$ and $14.5 \mathrm{mg}$ aminopyridinyl-derivative 6-DPPAP $\left(4.01 \cdot 10^{-2} \mathrm{mmol}, 1.0\right.$ eq. $)$ in $0.4 \mathrm{ml} \mathrm{CDCl}_{3}$. The formation of the heterodimeric complex $\left[\right.$ cis- $\left.\mathrm{PtCl}_{2}(\mathbf{6 a})(6-\mathrm{DPPAP})\right]$ was monitored by ${ }^{31} \mathrm{P}-$ NMR

${ }^{31}$ P-NMR (121 MHz, $\left.\mathrm{CDCl}_{3}, 293 \mathrm{~K}\right): \delta 107.5\left(\mathrm{dd}, J_{\mathrm{P}, \mathrm{Pt}}=4994.9, J_{\mathrm{P}, \mathrm{P}}=15.0 \mathrm{~Hz}\right), 13.9$ (br d, $\left.J_{\mathrm{P}, \mathrm{Pt}}=3555.1 \mathrm{~Hz}\right)\left(J_{\mathrm{P}, \mathrm{P}}\right.$-coupling not resolved due to broad line width).
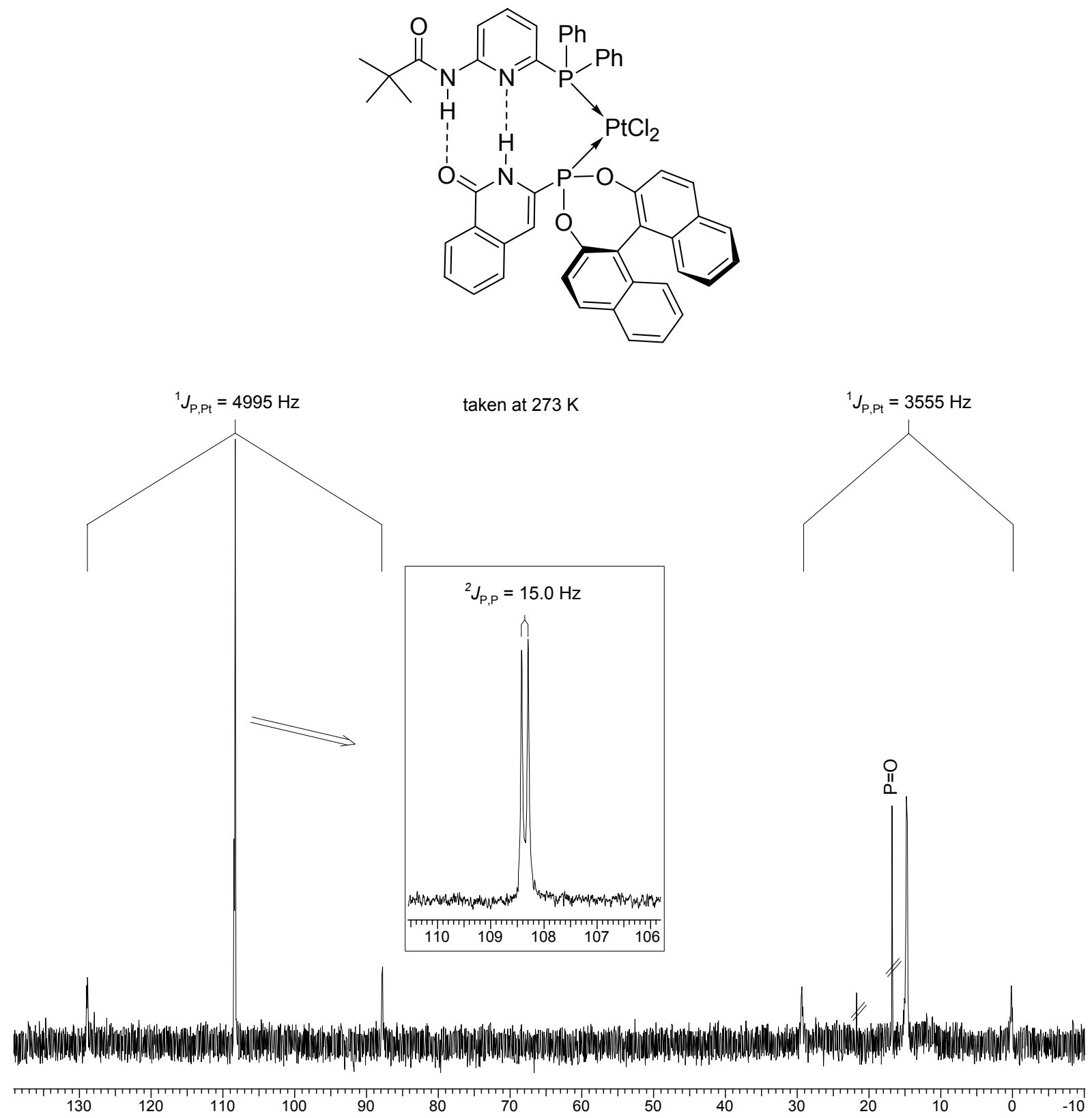
To a solution of $9.1 \mathrm{mg}\left[\mathrm{cis}-\mathrm{PtCl}_{2}(1,5-\mathrm{COD})\right]\left(2.43 \cdot 10^{-2} \mathrm{mmol}, 1.0\right.$ eq. $)$ in $0.4 \mathrm{ml} \mathrm{CDCl}_{3}$ was added a solution of $11.2 \mathrm{mg}$ isoquinolone $(R)-6 \mathbf{a}\left(2.43 \cdot 10^{-2} \mathrm{mmol}, 1.0\right.$ eq. $)$ and $10.0 \mathrm{mg}$ aminopyridinyl-derivative (-)-4a $\left(2.43 \cdot 10^{-2} \mathrm{mmol}, 1.0\right.$ eq. $)$ in $0.4 \mathrm{ml} \mathrm{CDCl}_{3}$. The formation of the heterodimeric complex $\left[c i s-\mathrm{PtCl}_{2}(\mathbf{6 a})(\mathbf{4 a})\right]$ was monitored by ${ }^{31} \mathrm{P}-\mathrm{NMR}$.

${ }^{31}$ P-NMR $\left(202 \mathrm{MHz}, \mathrm{CDCl}_{3}, 320 \mathrm{~K}\right): \delta 106.4\left(\mathrm{dd}, J_{\mathrm{P}, \mathrm{Pt}}=5020.8, J_{\mathrm{P}, \mathrm{P}}=12.3 \mathrm{~Hz}\right), 12.6(\mathrm{br} \mathrm{d}$, $\left.J_{\mathrm{P}, \mathrm{Pt}}=3617.9 \mathrm{~Hz}\right)\left(J_{\mathrm{P}, \mathrm{P}}\right.$-coupling not resolved due to broad line width).
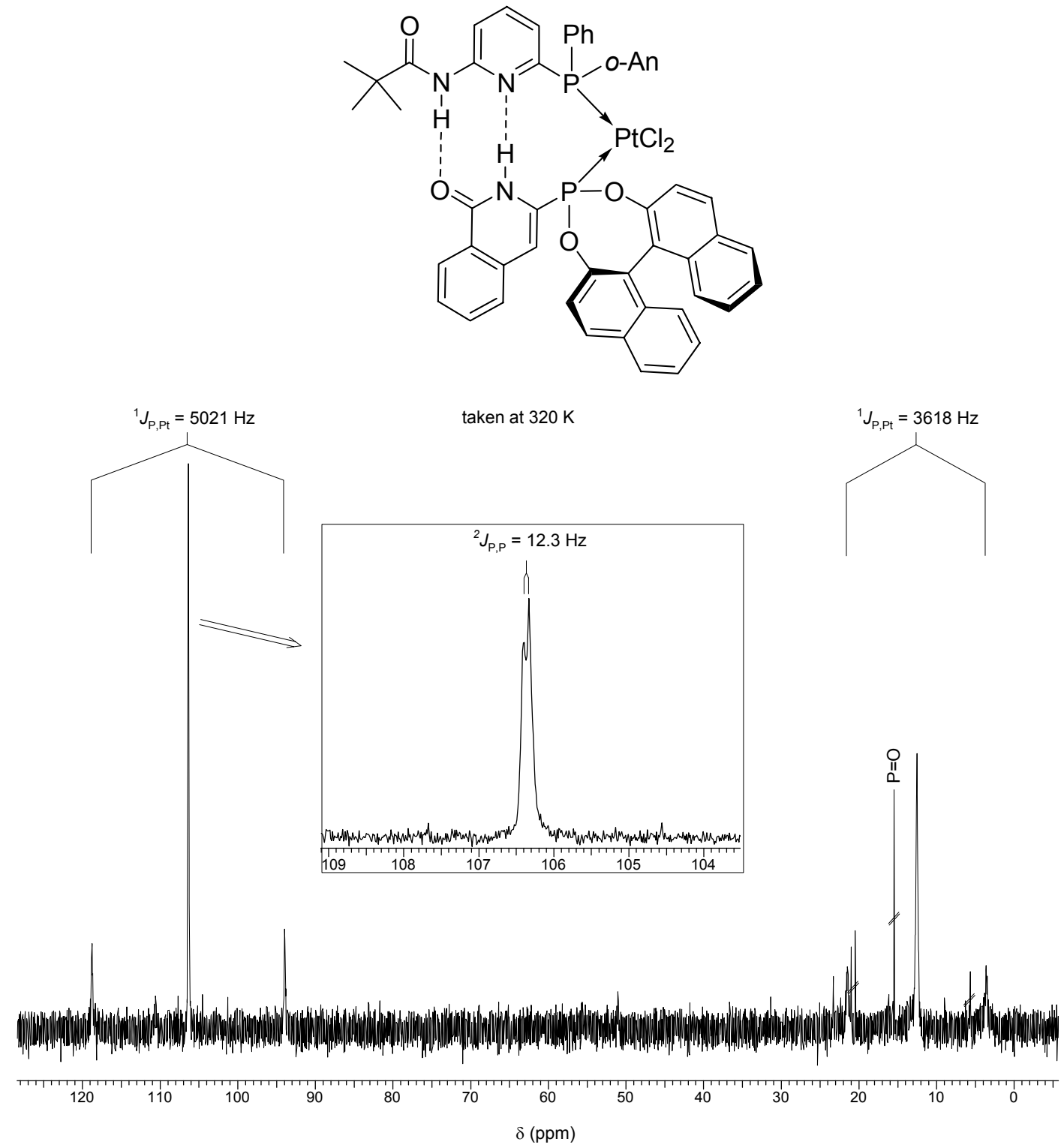


\section{Heterodimeric cationic rhodium complexes}

\section{Rhodium complex of $(S)$-5a and 3-DPICon:}

To a solution of $10.9 \mathrm{mg}\left[\mathrm{Rh}(1,5-\mathrm{COD})_{2}\right] \mathrm{BF}_{4}\left(2.68 \cdot 10^{-2} \mathrm{mmol}, 1.0\right.$ eq. $)$ in $0.4 \mathrm{ml} \mathrm{CDCl}_{3}$ was added a solution of $8.8 \mathrm{mg} 3$-DPICon $\left(2.67 \cdot 10^{-2} \mathrm{mmol}, 1.0\right.$ eq.) and $13.2 \mathrm{mg}$ aminopyridinylderivative $(S)-5 \mathbf{a}\left(2.68 \cdot 10^{-2} \mathrm{mmol}, 1.0\right.$ eq. $)$ in $0.4 \mathrm{ml} \mathrm{CDCl}_{3}$. The formation of the heterodimeric complex $[\mathrm{Rh}(1,5-\mathrm{COD})(\mathbf{5 a})(6-\mathrm{DPIC}$ ) $)]$ was monitored by ${ }^{31} \mathrm{P}-\mathrm{NMR}$ and mass spectrometry.

${ }^{31}$ P-NMR (162 MHz, $\left.\mathrm{CDCl}_{3}, 293 \mathrm{~K}\right): \delta 162.8\left(\mathrm{dd}, J_{\mathrm{P}, \mathrm{Rh}}=218.0 \mathrm{~Hz}, J_{\mathrm{P}, \mathrm{P}}=35.7 \mathrm{~Hz}\right), 154.7(\mathrm{~d}$, $\left.J_{\mathrm{P}, \mathrm{Rh}}=216.0 \mathrm{~Hz}\right), 33.9\left(\mathrm{br} \mathrm{d}, J_{\mathrm{P}, \mathrm{Rh}}=150.6 \mathrm{~Hz}\right)\left(J_{\mathrm{P}, \mathrm{P}-\text { coupling not resolved due to broad line }}\right.$ width).

MS (ESI; $\left.\mathrm{C}_{59} \mathrm{H}_{53} \mathrm{BF}_{4} \mathrm{~N}_{3} \mathrm{O}_{4} \mathrm{P}_{2} \mathrm{Rh}, \mathrm{M}=1119.26 \mathrm{~g} / \mathrm{mol}\right): \mathrm{m} / \mathrm{z}=622$ (27), 703 (48), 884 (11), 924 $\left(\mathrm{M}^{+}-\mathrm{COD}-\mathrm{BF}_{4}^{-}, 100\right), 1087$ (32).

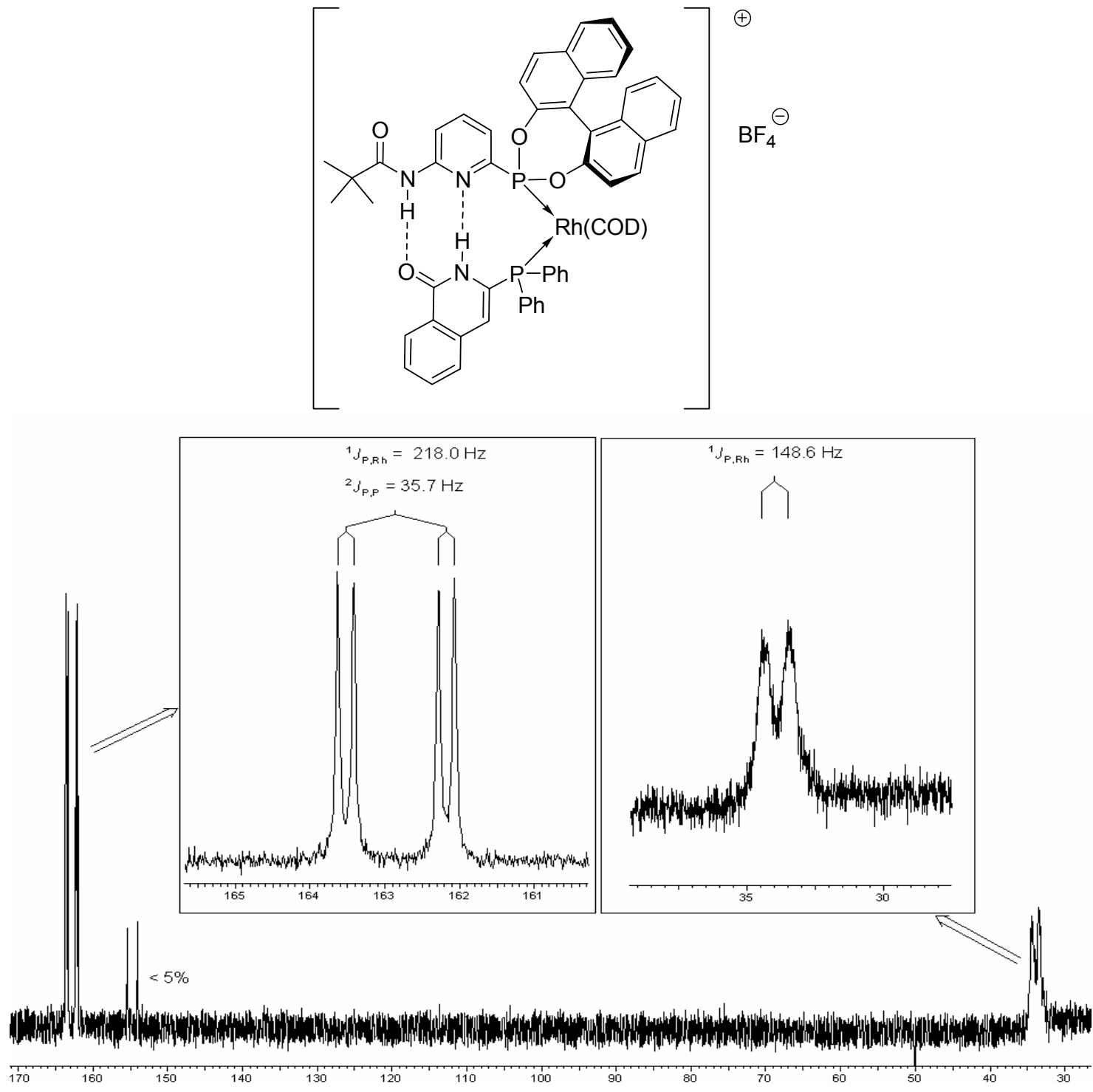




\section{Rhodium complex of (S)-5a and (S)-6a:}

Mass spectrometry (ESI) proved the formation of the heterodimeric complex $\left(\mathrm{M}^{+}-\mathrm{BF}_{4}{ }^{-}=\right.$ 1162 (24), $\mathrm{M}^{+}-\mathrm{COD}-\mathrm{BF}_{4}{ }^{-}=1054$ (100)). However the ${ }^{31} \mathrm{P}-\mathrm{NMR}$ showed a broad signal at a chemical shift of $\delta=157 \mathrm{ppm}$ which confirms phosphonite ligand coordinated to a rhodium(I) center. ${ }^{[10]}$ The broad line width, which is a consequence of an unresolved dynamic process, did in this case not allow an unambigeous structural assignment. However, replacing COD by addition of the bidentate ligand 1,10-phenanthroline gave the defined complex [Rh(5a)(6a)phen] $\mathrm{BF}_{4}$ described below. The ${ }^{31} \mathrm{P}-\mathrm{NMR}$ of $[\mathrm{Rh}(\mathbf{5 a})(\mathbf{6 a})$ phen]BF 4 at $270 \mathrm{~K}$ shows two ABX spin systems in accord with two diastereomeric heterodimer rhodium complexes in a ratio of 1.5:1.

The formation of diastereomers may result either from a distorted square planar coordination geometry at rhodium ${ }^{[11]}$ or alternatively, may be due to additional axial chirality as a consequence of a distorsion in the hydrogen bond system of the ligand backbone (compare Xray structure of a square planar platinum(II) complex of the parent heterodimer ligand system 6-DPPAP/3-DPICon published in ref. [5] in the communication).

\section{Rhodium complex of $(S)-5$ a and $(S)$-6a in the presence of 1,10-phenanthroline:}

To a solution of $10.9 \mathrm{mg}\left[\mathrm{Rh}(1,5-\mathrm{COD})_{2}\right] \mathrm{BF}_{4}\left(2.68 \cdot 10^{-2} \mathrm{mmol}, 1.0\right.$ eq. $)$ in $0.4 \mathrm{ml} \mathrm{CDCl}_{3}$ was added a solution of $13.2 \mathrm{mg}$ aminopyridinyl-derivative $(S)-5 \mathbf{a}\left(2.68 \cdot 10^{-2} \mathrm{mmol}, 1.0 \mathrm{eq}.\right)$ and $12.3 \mathrm{mg}$ isoquinolone $(S)-6 \mathbf{a}\left(2.68 \cdot 10^{-2} \mathrm{mmol}, 1.0\right.$ eq.) in $0.4 \mathrm{ml} \mathrm{CDCl}_{3}$. After dissolution of the complex, $4.8 \mathrm{mg} 1,10$-phenanthroline $\left(2.66 \cdot 10^{-2} \mathrm{mmol}, 1.0 \mathrm{eq}\right.$.) was added causing the solution to turn red immediately. The formation of the heterodimeric complex [Rh(5a)(6a)phen]BF 4 was monitored by ${ }^{31} \mathrm{P}-\mathrm{NMR}$ and mass spectrometry.

${ }^{31}$ P-NMR (202 MHz, $\left.\mathrm{CDCl}_{3}, 270 \mathrm{~K}\right): \delta 181.0\left(\mathrm{dd}, J_{\mathrm{P}, \mathrm{Rh}}=235.6 \mathrm{~Hz}, J_{\mathrm{P}, \mathrm{P}}=85.8 \mathrm{~Hz}\right), 178.4(\mathrm{~d}$, $\left.J_{\mathrm{P}, \mathrm{Rh}}=246.1 .0 \mathrm{~Hz}\right), 177.9\left(\mathrm{dd}, J_{\mathrm{P}, \mathrm{Rh}}=241.2 \mathrm{~Hz}, J_{\mathrm{P}, \mathrm{P}}=92.3 \mathrm{~Hz}\right), 165.1\left(\mathrm{dd}, J_{\mathrm{P}, \mathrm{Rh}}=240.4 \mathrm{~Hz}\right.$, $\left.J_{\mathrm{P}, \mathrm{P}}=85.9 \mathrm{~Hz}\right), 164.4\left(\mathrm{dd}, J_{\mathrm{P}, \mathrm{Rh}}=229.6 \mathrm{~Hz}, J_{\mathrm{P}, \mathrm{P}}=92.3 \mathrm{~Hz}\right)$.

MS (ESI; $\left.\mathrm{C}_{71} \mathrm{H}_{51} \mathrm{BF}_{4} \mathrm{~N}_{5} \mathrm{O}_{6} \mathrm{P}_{2} \mathrm{Rh}, \mathrm{M}=1321.24 \mathrm{~g} / \mathrm{mol}\right): \mathrm{m} / \mathrm{z}=1201$ (5), $1236\left(\mathrm{M}^{+}-\mathrm{BF}_{4}^{-}, 100\right)$, 1027 (35), 1307 (5). 

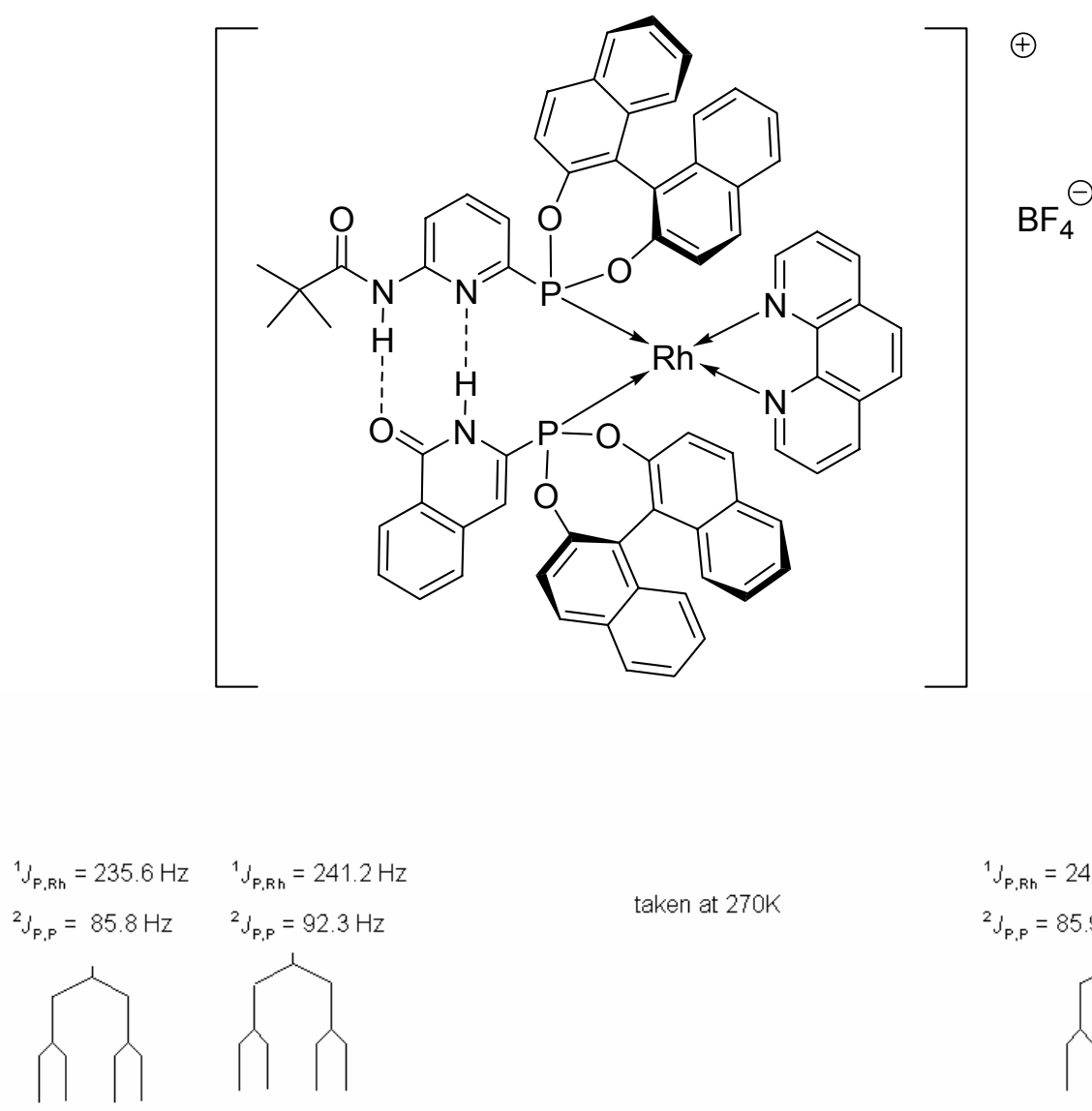

taken at $270 \mathrm{~K}$

${ }^{1} J_{\mathrm{P}, \mathrm{Rh}}=240.4 \mathrm{~Hz}{ }^{1} J_{\mathrm{P}, \mathrm{Rh}}=229.6 \mathrm{~Hz}$

${ }^{2} J_{P, P}=85.9 \mathrm{~Hz} \quad{ }^{2} J_{P, P}=92.4 \mathrm{~Hz}$
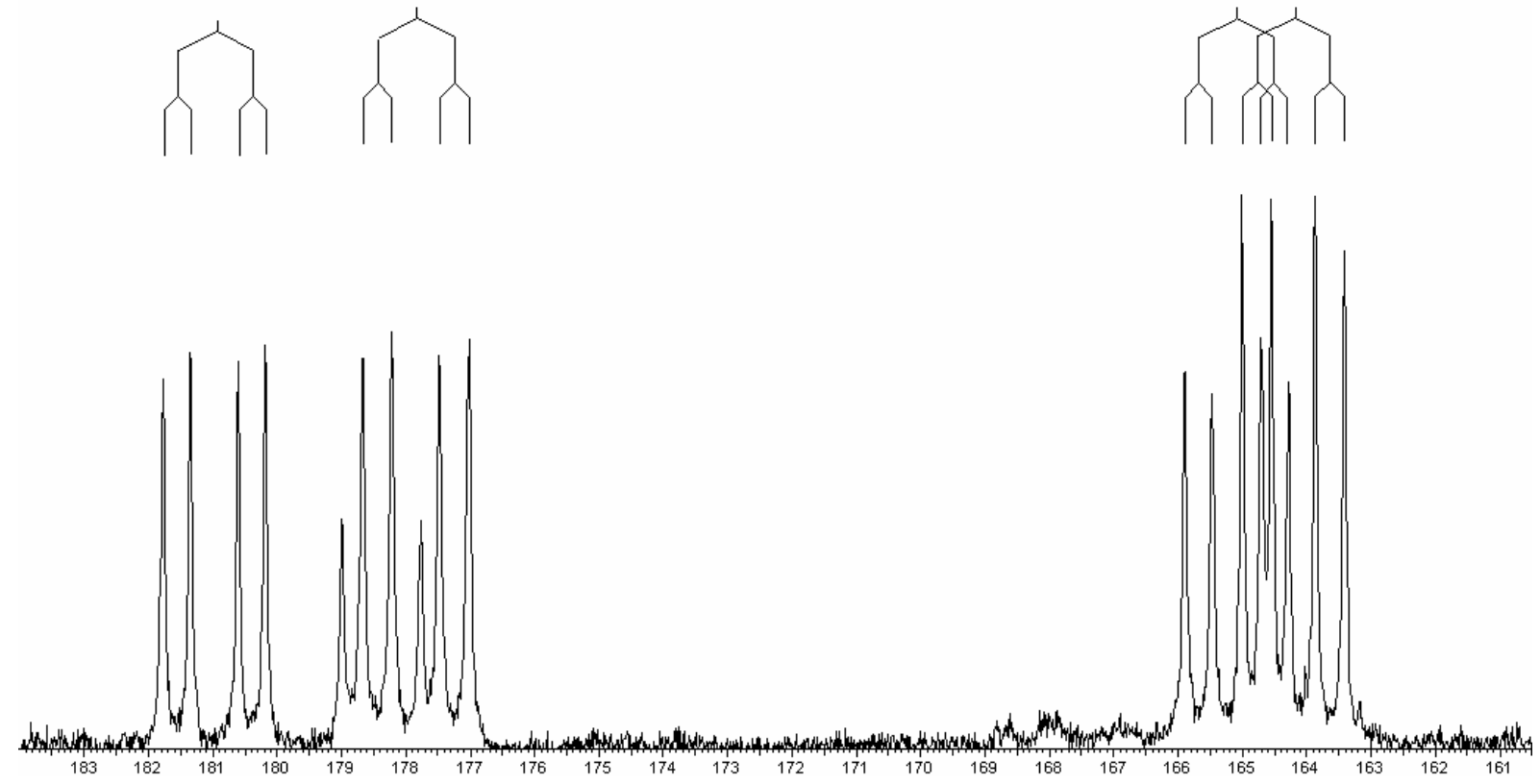
Rhodium complex of $(S)$-5b and $(S)$-6a in the presence of 1,10-phenanthroline:

Mass spectrometry (ESI) and ${ }^{31} \mathrm{P}-\mathrm{NMR}$ prove the formation of the heterodimeric complex. In agreement with the spectrum shown above, two diastereomeric heterodimer rhodium complexes in a ratio of 1.5:1 were observed which result again from a distorted square planar geometry or supplementary axial chirality in the hydrogen bonded ligand backbone.

To a solution of $10.9 \mathrm{mg}\left[\mathrm{Rh}(1,5-\mathrm{COD})_{2}\right] \mathrm{BF}_{4}\left(2.68 \cdot 10^{-2} \mathrm{mmol}, 1.0\right.$ eq. $)$ in $0.4 \mathrm{ml} \mathrm{CDCl}_{3}$ was added a solution of $14.0 \mathrm{mg}$ aminopyridinyl-derivative $(S)-5 \mathbf{b}\left(2.69 \cdot 10^{-2} \mathrm{mmol}, 1.0 \mathrm{eq}.\right)$ and $12.3 \mathrm{mg}$ isoquinolone $(S)-\mathbf{6 a}\left(2.68 \cdot 10^{-2} \mathrm{mmol}, 1.0 \mathrm{eq}\right.$.) in $0.4 \mathrm{ml} \mathrm{CDCl}_{3}$. After dissolution of the complex, $4.8 \mathrm{mg} 1,10$-phenanthroline $\left(2.66 \cdot 10^{-2} \mathrm{mmol}, 1.0 \mathrm{eq}\right.$.) was added causing the solution to turn red immediately. The formation of the heterodimeric complex $[\mathrm{Rh}(\mathbf{5 b})(\mathbf{6 a})$ phen $] \mathrm{BF}_{4}$ was monitored by ${ }^{31} \mathrm{P}-\mathrm{NMR}$ and mass spectrometry.

${ }^{31}$ P-NMR $\left(162 \mathrm{MHz}, \mathrm{CDCl}_{3}, 270 \mathrm{~K}\right): \delta 180.5\left(\mathrm{dd}, J_{\mathrm{P}, \mathrm{Rh}}=235.5 \mathrm{~Hz}, J_{\mathrm{P}, \mathrm{P}}=86.7 \mathrm{~Hz}\right), 180.2$ $\left(\mathrm{dd}, J_{\mathrm{P}, \mathrm{Rh}}=242.7 \mathrm{~Hz}, J_{\mathrm{P}, \mathrm{P}}=91.3 \mathrm{~Hz}\right), 165.3\left(\mathrm{dd}, J_{\mathrm{P}, \mathrm{Rh}}=242.5 \mathrm{~Hz}, J_{\mathrm{P}, \mathrm{P}}=87.0 \mathrm{~Hz}\right), 159.5(\mathrm{dd}$, $\left.J_{\mathrm{P}, \mathrm{Rh}}=227.5 \mathrm{~Hz}, J_{\mathrm{P}, \mathrm{P}}=89.3 \mathrm{~Hz}\right)$.

MS (ESI; $\left.\mathrm{C}_{73} \mathrm{H}_{55} \mathrm{BF}_{4} \mathrm{~N}_{5} \mathrm{O}_{6} \mathrm{P}_{2} \mathrm{Rh}, \mathrm{M}=1349.27 \mathrm{~g} / \mathrm{mol}\right): \mathrm{m} / \mathrm{z}=1082\left(\mathrm{M}^{+}\right.$-phen- $\left.\mathrm{BF}_{4}^{-}, 100\right)$, 1143 (78), $1262\left(\mathrm{M}^{+}-\mathrm{BF}_{4}^{-}, 59\right)$.

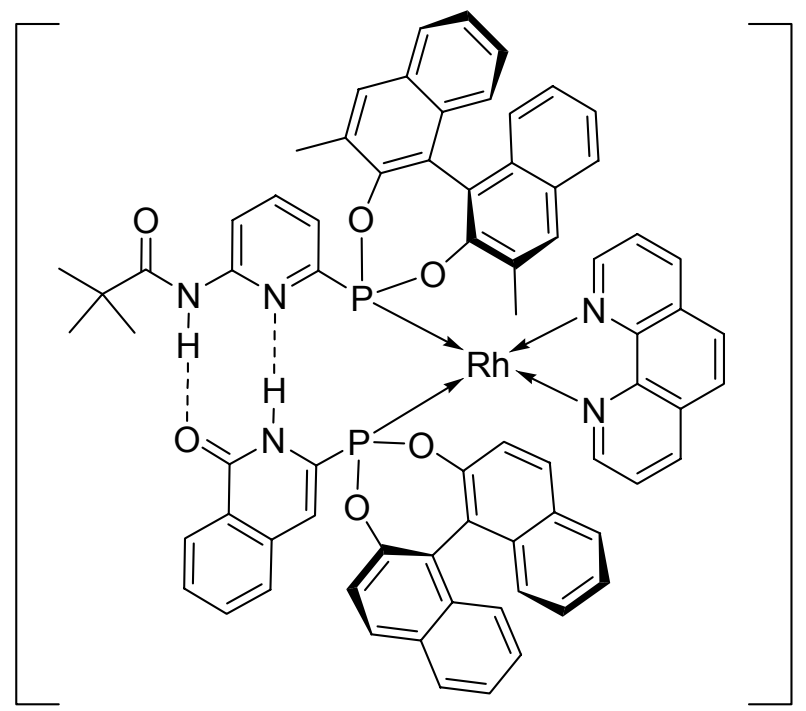

$\oplus$

$\mathrm{BF}_{4}^{\ominus}$ 


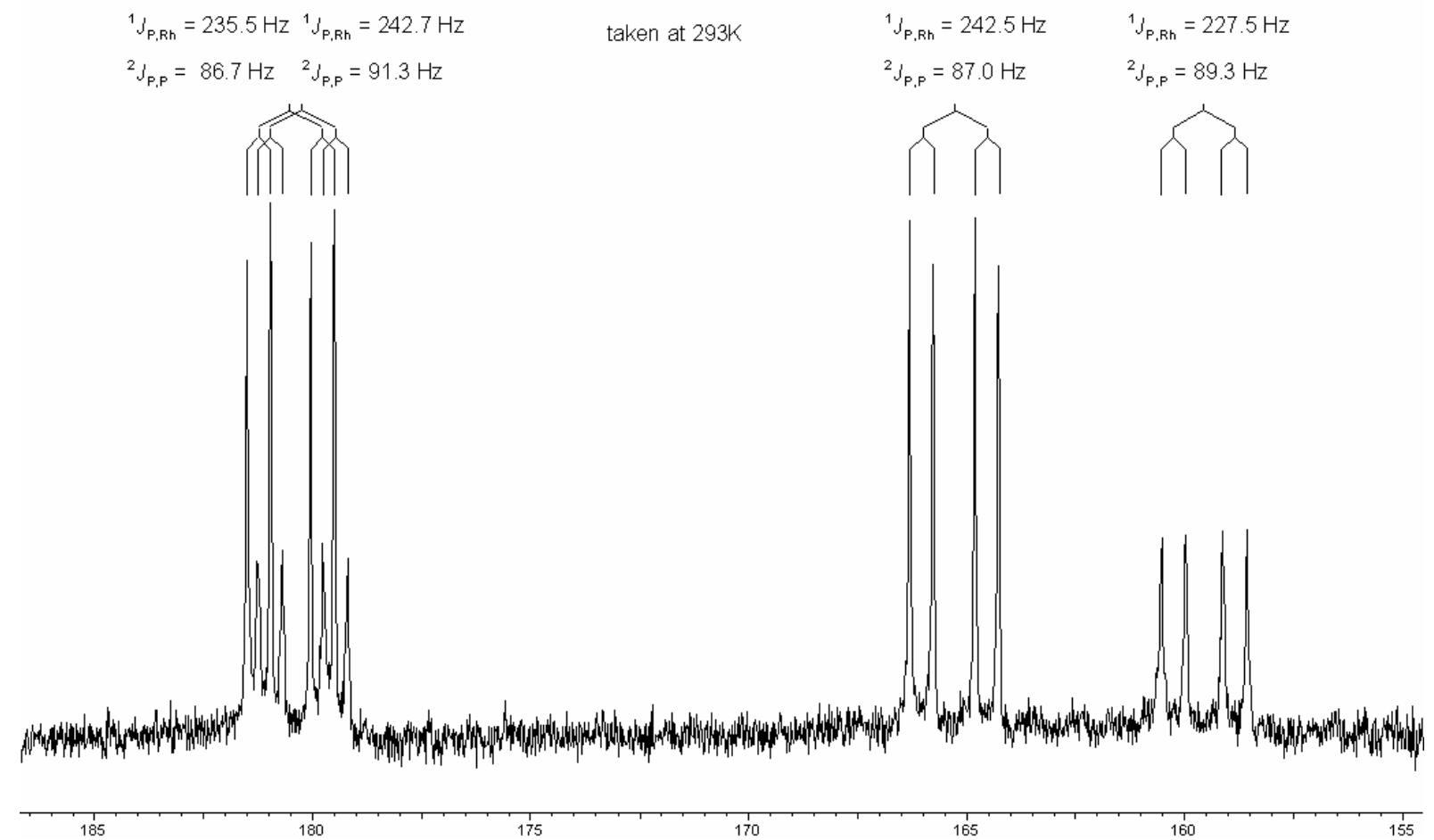


[1] Sakai, J.; Schweizer, W.B.; Seebach, D. Helv. Chim. Acta. 1993, 76, 2654-2665.

[2] Davis, D. D.; Jacocks, H. M. J. Organomet. Chem. 1981, 206, 33-47.

[3] Simonsen, K. B.; Gothelf, K. V.; Jørgensen J. Org. Chem. 1998, 63, 7536-7538 and references cited therein.

[4] Den Hertog, H. J, Wibaut, J. P. Recl. Trav. Chim. Pays 1932, 51, 381-387 and ibid. 1936, 55, 122-125.

[5] Mase, T. et al. J. Org. Chem. 2001, 66, 6775-6786.

[6] Osborn, A. R.; Schofield, K.; Short, L. N. J. Chem. Soc. 1956, 4191-4206.

[7] (a) Wittig, G.; Braun, H.; Cristau H. J. Justus Liebigs Ann. Chem. 1971, 751, 17-26.

(b) Chodkiewicz, W.; Guillerm, D.; Jore, D.; Mathieu, E.; Wodzki, W. J. Organomet. Chem. 1984, 269, 107-114.

[8] Gladiali, S.; Pinna L. Tetrahedron Asym. 1991, 2, 623-632.

[9] Still, W. C.; Kahn, M.; Mitra, A. J. Org. Chem. 1978, 43, 2923-2925.

[10] Van der Vlugt, J. I., Paulusse J. M. J., Zijp E. J., Tijmensen J. A., Mills A. M., Spek A. L., Claver C., Vogt D. Eur. J. Inorg. Chem. 2004, 4193-4201.

[11] (a) Jones R. A. et al. J. Chem. Soc. Dalton Trans. 1979, 489-490. [Rh( $\left.\left(\mathrm{PMe}_{3}\right)_{4}\right]^{+}$has a tetraedral coordinative geometry.

(b) Caldaran H., DeArmond, M. K., Hanck K. W., Sahini, V. E. J. Am. Chem. Soc. 1976, 98, 4455-57. $\left[\mathrm{Rh}(\mathrm{phen})_{2}\right]^{+}$has a distorted square planar structure. 